\title{
Die Schönheit der Vernunft - (Ir-)Rationalität von Rechtswissenschaft in Mittelalter und Moderne"
}

\author{
Helge Dedek**
}

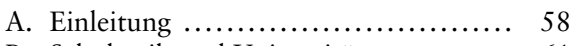

B. Scholastik und Universität .......... 61

I. Scholastik im Spiegel des

Humanismus

II. „Wissenschaft in der Gestalt der Schule"

III. Text, Autorität, ratio scripta ....... 63

IV. Schul-Unterricht ................. 66

C. Scholastik als Stil und „mentaler

Habitus“ 68

I. „Rechtsästhetik“? .................. 68

II. (Rechts-)Ästhetik im Mittelalter .... 70

1. Der ,italienische Stil“.......... 70
2. Scholastische „Ästhetik“? ....... 71

3. Erwin Panofsky: Scholastik als „mentaler Habitus“ ........... 73

4. Scholastische „Ästhetik“: Harmonie und Ordnung ..................... 75

D. Scholastik am Werk: Die

Interessendoktrin .................. 76

I. Begriffsbildung und Distinktion und ein „neuzeitlicher“ Exzess...... 77

II. Bartolus: Formvollendung und „Zweckrationalität“................ 80

E. Schluss .......................... 82

\section{A. Einleitung}

Die westeuropäische Rechtstradition ${ }^{1}$ - und insbesondere die des kontinentalen $\mathrm{Zi}$ vilrechts - versteht sich als Tradition rationaler Rechtsfindung und wissenschaftlicher Behandlung von Recht. Der Begriff der Tradition impliziert Kontinuität. Er evoziert zudem die Vorstellung von Entwicklung. Im Sinne einer solchen Entwicklung kann etwa Max Webers Beschreibung des Wegs der Rechtsfindung hin zu einer „formalen Rationalität“2 gelesen werden, die sich als eingebettet in ein Modell der kontinuierlichen gesellschaftlichen Gesamtentwicklung hin zu einem höheren

* Der vorliegende Aufsatz ist die etwa um die Hälfte gekürzte und ins Deutsche übertragene Fassung einer Abhandlung, die im Oktober 2008 bei dem Harvard Stanford International Junior Faculty Forum an der Stanford Law School vorgestellt wurde. Für die hilfreichen Kommentare und die Einladung schulde ich Dank vor allem den Herren Professoren Lawrence M. Friedman und Richard T. Ford (Stanford) sowie William P. Alford (Harvard). Bei einem Vortrag anlässlich des „Forums Junge Rechtswissenschaft" an der Eberhard-Karls-Universität Tübingen haben mir vor allem die Herren Professoren Knut-Wolfgang Nörr, Gottfried Schiemann und Martin Gebauer äußerst wertvolle Hinweise gegeben. Für hilfreiche Hinweise danke ich zudem den Herren Professoren H. Patrick Glenn, Nicholas Kasirer, Desmond Manderson (McGill) und Martin J. Schermaier (Rheinische FriedrichWilhelms-Universität Bonn). Quellen hatte ich eingesehen im Institut für Römisches Recht und Vergleichende Rechtsgeschichte der Rheinischen Friedrich-Wilhelms-Universität Bonn. - Ganz überwiegend zitiert wird englischsprachiges Schrifttum, auch in Bereichen, in denen reiche deutsche (italienische, etc.) Literatur existiert. Dies ist der Genese des Texts geschuldet, der sich ursprünglich an ein amerikanisches Publikum wendete. Seit mich die Bitte der Herausgeber erreichte, eine Übersetzung anzufertigen, konnte ich den Text nicht mehr umfassend umarbeiten; leider hatte ich auch keinen Zugang zu einer deutschen Bibliothek. Ich bitte den geneigten Leser um Verständnis

* Prof. Dr. Helge Dedek, LL.M. (Harvard) ist Assistant Professor an der McGill University, Faculty of Law in Montreal, Kanada.

1 Siehe etwa H. J. Berman, Law and Revolution [:] The Formation of the Western Legal Tradition, Cambridge, Mass.: Harvard University Press 1983, S. 1 ff.

2 M. Weber, Wirtschaft und Gesellschaft, 5. Aufl., Tübingen 1972, S. 395 ff. Vgl. dazu etwa D. Kenne$d y$, The Disenchantment of Logically Formal Legal Rationality, or Max Weber's Sociology of the Contemporary Mode of Western Legal Thought, Hastings L.J. 55 (2003-2004), S. 1031. 
Grad rationaler Durchdringung und Organisation deuten lässt. ${ }^{3}$ Zugleich sind wir gewohnt, (Rechts-)Geschichte in Epochen zu denken. ${ }^{4}$ Periodisierung von Geschichte betont Differenz und Umbruch gegenüber Kohärenz und Kontinuität: Zeiträume werden gegen andere abgegrenzt, um einen bestimmten „Zeitgeist“, um bestimmte Merkmale einer Epoche ex post zu erfassen und zu benennen. ${ }^{5}$ Epochen, die mit „Rationalität“ und „Vernunft“ assoziiert werden, sind etwa Aufklärung und Renaissance - das „Mittelalter“ hingegen, als „medium aevum“ gleichsam ungeschlachter Lückenfüller zwischen Antike und „Neuzeit“, ist durch die taxonomische Grenze, die Heraufkunft der „Moderne“, von unserem intellektuellem Zugang abgeschnitten: Mit dem Beginn der Moderne beginnt auch eine uns erschließbare, vom Individuum ausgehende Erfahrungswelt. Das Mittelalter hingegen, qua definitionem „prae-modern“, 6 findet sich auf der anderen Seite der Demarkationslinie, die den modernen, rationalen Verstand, die uns von dem Mensch des Mittelalters trennt. ${ }^{7}$

Diese (sicherlich zumeist nur unterbewusst vorgenommene) Kategorisierung äußert sich auch im rechtshistorischen Kontext. Die „Renaissance“ des römischen Rechts ist zwar fraglos conditio sine qua non für die sich in Kontinentaleuropa entfaltende Entwicklung hin zur „formalen Rationalität“. Materielle Kontinuität findet indes traditionell weniger Beachtung. Die Rückbesinnung der Historischen Schule zielte auf das Freilegen der klassischen Quellen - Savigny selbst schuf zwar mit seiner „Geschichte des römischen Rechts im Mittelalter“ ein Standardwerk zur mittelalterlichen Jurisprudenz, zugleich aber wandten sich die dogmatischen Schriften der Zeit ab von dem Diskurs des ius commune und der bartolistischen Tradition, auf dem er beruhte. Die Behandlung der Quellen im Mittelalter erschien und erscheint dem modernen Beobachter prima facie erratisch, bisweilen geprägt von fast kindlichem Formalismus, irrational oder zumindest geleitet von einer Rationalität, die uns unzugänglich erscheint. Als Synekdoche für diese uns scheinbar fremde Denkweise steht oft - zumindest im untechnischen Sprachgebrauch - die Bezeichnung als „scholastisch”. Wie das Wort „Mittelalter“ selbst hat die Bezeichnung „scholastisch“ oft

3 J. Elster, Rationality, Economy and Society, in: S. Turner (Hrsg.), The Cambridge Companion to Weber, Cambridge University Press 2000, S. 21.

4 C. B. McCullagh, Colligation and Classification in History, History and Theory 17 (1978) S. $267 \mathrm{ff}$. Vgl. zu diesem Spannungsverhältnis etwa auch M. Foucault, L'Archéologie du savoir, Paris: Gallimard, S. $33 \mathrm{ff}$.

5 Zu einem solchen Verständnis von Rechtsgeschichte etwa F. Wieacker, Privatrechtsgeschichte der Neuzeit, 2. Aufl., Göttingen 1967, S. 20 ff.; zum „Kontinuitätsbegriff“ S. 43 ff.

6 L. Patterson, On the Margin - Postmodernism, Ironic History, and Medieval Studies, Speculum 65 (1990), S. 87 (92).

7 M. de Grazia, The Modern Divide - From Either Side, Journal of Medieval and Early Modern Studies 37 (2007), S. 453: „No period division has had more consequence than the divide between the ,medieval and the ,modern', with the exception, perhaps, of that between b.c. and a.d.". 
einen abwertenden Beiklang, und findet sich im (rechts-)wissenschaftlichen Diskurs etwa dann, wenn geistlose Begriffsakrobatik bemäkelt wird. ${ }^{8}$

Allein die - in der Tat und im technischen Sinne - scholastische Jurisprudenz ${ }^{9}$ der mittelalterlichen Romanisten und Kanonisten ist nicht so weit entfernt, wie der erste Eindruck glauben machen mag. In ihrer Hochzeit sah sich die scholastische Methode als der Inbegriff angewandter ratio, die manchen in ihrer Fühllosigkeit für das Mystische und kalten Rationalität bisweilen sogar lästerlich erschien. ${ }^{10}$ Zugleich gab die Scholastik sich bisweilen einer eigentümlichen, „irrationalen“ Manieriertheit hin. In beiderlei Hinsicht fällt uns, um noch einmal mit Weber zu sprechen, das „Nacherleben " 11 schwer. Das intellektuelle Nachvollziehen wird uns erleichtert, wenn wir uns die Prämissen der scholastischen Methode und vor allem ihre Wurzeln in der Pädagogik, der mittelalterlichen Schul-Bildung in Erinnerung rufen (Teil B). Doch soll unser Hauptaugenmerk nicht einer „Rehabilitierung “ der Rationalität scholastischer Jurisprudenz gelten, derer es, wie wir sehen werden, gar nicht bedarf. Wir wollen demgegenüber anschließend versuchen, bewusst die irrationalen Aspekte des scholastischen „Habitus“ ein wenig näher zu beleuchten, jene Aspekte, die sich einer (zweck-)rationalen Erkärung entziehen (Teil C). Wir haben eingangs von Recht als Tradition gesprochen. Traditionales Handeln ist indes nie allein mit rationalen Kriterien erklärbar. ${ }^{12}$ Die dem traditionalen Handeln strukturell inhärente Irrationalität führt freilich dort zu einer paradoxen Spannung, wo sich eine Tradition selbst als Tradition von Rationalität definiert - wie es die westliche Rechtswissenschaft traditionell tut. ${ }^{13}$ Am Beispiel der scholastischen Jurisprudenz wird besonders deutlich, wie eine Tradition - von Wissenschaft, von Ausbildung, etc. - auch die Produktion juristischer Texte beeinflusst, ohne dass sich alle Eigenheiten solcher Textproduktion durch einen bestimmten Zweck rational rechtfertigen ließen. Das Verständnis dieser

8 J. Haldane, Scholasticism - Old and New, The Philosophical Quarterly 43 (1993) S. 403 ff. Als Beispiel für die Handhabung im pejorativen Sinne siehe etwa in der jüngeren Vergangenheit R. H. Pildes, Forms of Formalism, U. Chi. L. Rev. 66 (1999), S. 607 (610); D V. Snyder, The Law of Contract and the Concept of Change - Public and Private Attempts to Regulate Modification, Waiver, and Estoppel, Wis. L. Rev. 1999, S. 607 (621); J. LaPalombara, Politcal Power and Political Development, Yale L.J. 78 (1968-1969) S. 1253 (1257).

9 Jüngst, statt aller, S. Lepsius, Von Zweifeln zur Überzeugung, Frankfurt a.M. 2003, S. $201 \mathrm{ff}$. Dort auch ausführlich, e.g., zur Rolle der „Rationalität“ im Kontext des Zeugenbeweises bei Bartolus, S. $83 \mathrm{ff}$.

10 Man denke nur an Abaelard und die gegen ihn gerichtete Kampagne des H1. Bernhard von Clairvaux (zu der Spannung zwischen Vernunft und Glaube siehe etwa C. J. Mews, Philosophy and Theology 1100-1150 - The Search for Harmony, in: ders. (Hrsg.), Reason and Belief in the Age of Roscelin and Abelard.Aldershot: Ashgate 2002, S. 159 ff. Bemerkenwert Im Hinblick auf die innere Verbindung der ratio mit dem trivium - Grammatik, Rhetorik, Logik - ist die von Petrus Damiani überlieferte Bemerkung, der erste Grammatiker sei niemand anderes als Satan selbst gewesen.

11 Weber, Wirtschaft und Gesellschaft (Fn. 2), S. 2.

12 Ibid., S. 12. Siehe dazu und zur „unthinkingness“ von Tradition L. J. Munoz, The Rationality of Tradition, Archiv für Rechts- und Sozialphilosophie 67 (1981), S. 197 (201ff.).

13 H.P. Glenn, Legal Traditions of the World, 3. Aufl., Oxford University Press 2007, S. 19. Siehe im übrigen die Zusammenfassung unten D. 
notwendig „irrationalen“ Komponente juristischen Diskurses mag uns, den Modernen, die eigene - oft nicht eingestandene - Irrationalität ein wenig näher bringen und helfen, den „cult of reason“ 14 , in dem wir befangen sind, ein wenig kritischer zu betrachten.

\section{B. Scholastik und Universität}

\section{Scholastik im Spiegel des Humanismus}

Das Phänomen der Scholastik wird am besten verständlich, wenn man es weniger mit Inhalten - etwa thomistischer Philosophie ${ }^{15}$ - denn mit einer Vorgehensweise identifiziert. Der Titel der noch stets autoritativen zweibändigen Abhandlung von Martin Grabmann weist den Weg: „Die Geschichte der scholastischen Methode“ zeigt die Scholastik als aus der Kultur der Schule hervorgegangene Denk- und Arbeitsweise mit einer Geschichte, die weit vor das Hochmittelalter zurückreicht. ${ }^{16}$ Es ist freilich just jener charakteristische modus operandi, das penible Abwägen der Autoritäten, die Distinktionen, Kategorienbildungen und Taxonomien, die der Scholastik ihren schlechten Ruf eingebracht haben. ${ }^{17}$

Wie fremdartig uns die scholastische Argumentations- und Denkweise oft vorkommen mag: Die unkritische Übernahme des intellektuellen Unwerturteils verbietet sich schon deshalb, weil es seinerseits aus einem spezifischen Kontext stammt, der die Interessen hinter der Polemik offenlegt. Es fand ein regelrechter „academic turf war"18 statt, die aufstrebenden Humanisten schrieben gegen das scholastische „establishment" an, ${ }^{19}$ das alle Universitätsdisziplinen beherrschte. ${ }^{20}$ Wie auch die Bezeichnung als „gotisch“ - sprich: deutsch - dezidiert als Verunglimpfung intendiert

14 G. Bagnall, Law as Art, Dartmouth Publishing Company 1996, S. 148.

15 Vgl. etwa M. L. Colish, Remapping Scholasticism, Pontifical Institute of Mediaeval Studies 2000, die sehr anschaulich die Bedeutung der religiösen/weltanschaulichen Ausrichtung des jeweiligen Betrachters auf die Darstellung der Scholastik darlegt. - Vor einer Gleichsetzung von Philosophie und Methode warnt etwa auch G. Otte, Die Aristoteleszitate in der Glosse, Beobachtungen zur philosophischen Vorbildung der Glossatoren, Zeitschrift der Savigny-Stiftung für Rechtsgeschichte Rom. Abt. 85 (1968), S. 368 (391), wenn er darlegt, dass Feststellungen über die „scholastische“ Arbeitsweise der Glossatoren nichts aussagt über die tatsächlichen philosophische Einflüsse, unter denen sie gestanden haben mögen.

16 M. Grabmann, Die Geschichte der scholastischen Methode nach den gedruckten und ungedruckten Quellen, Freiburg 1909.

17 Haldane, Scholasticism (Fn. 8), S. 404: „inward-looking, unproductive, academic hair-splitting. “

18 N.S. Amos, New Learning, old theology - Renaissance biblical humanism, scripture, and the question of theological method, Renaissance Studies 17 (2003), S. 39 (40).

19 C. Nauert, Humanism as Method - Roots of Conflict with the Scholastics, Sixteenth Century Journal 29 (1998), S. 427; ders., The Clash of Humanists and Scho1astics - An Approach to Pre-Reformation Controversies, Sixteenth Century Journal 4 (1973) S. 1.

20 Siehe C. A. Rubino, The Invisible Worm - Ancients and Moderns in „The Name of the Rose“, SubStance 14 (1985) S. 54 ff. 
war $^{21}$ - so wurde auch die Scholastik, ja alles „Mittelalterliche“ Opfer einer nachhaltig wirksamen Defamierung. ${ }^{22}$ Diese Nachwirkung bestand insbesondere darin, den Bruch, die Wende im Übergang von Scholastik und Humanismus übermäßig zu betonen und den Blick für Kontinuitäten zu trüben. ${ }^{23}$

\section{II. „Wissenschaft in der Gestalt der Schule“}

Die Leidenschaftlichkeit des Bildersturms deutet auf die Dominanz der Scholastik in der Akademie hin. Besonders deutlich wird dies in der Rechtswissenschaft, die sich im Kontext des Aufstiegs der mittelalterlichen Universität als Disziplin neu erfindet. Die Parameter der „Renaissance“ des römischen Rechts und der zeitgleichen Inauguration des juristischen studium in Oberitalien sind bekannt; ${ }^{24}$ beide Phänomene fallen in den weiteren Kontext der (mittlerweile immer weiter vorverlegten) „Renaissance des zwölften Jahrhunderts" ${ }^{25}$ : einer Zeit ökonomischen wie auch intellektuellen Aufschwungs ${ }^{26}$ wie auch des Aufstiegs der mittelalterlichen „Latinität“27. In diesem intellektuellen Klima findet der Neubeginn statt, der die kontinentale, universitäre Rechtstradition begründet: Die Wiege des kontinentalen Zivilrechts stand in Rom - in Bologna hingegen ging es zur Schule.

Scholastik ist, buchstäblich, die Methode und Kultur von Schul-Bildung. Diese umfasste zunächst das Studium der freien Künste. Der Zyklus des trivium war hierbei die methodische Grundlegung: Die drei Künste Grammatik, Rhetorik und Dialektik (Logik) lehrten den Schüler die rechte Art zu lesen, zu schreiben und zu denken. Jedwede intellektuelle Aktivität fand sodann im Rahmen der in diesem grundlegen-

21 Vgl. etwa M. Trachtenberg, Suger's Miracles, Branner's Bourges - Reflections on „Gothic Architecture" as Medieval Modernism, Gesta 39 (2000) S. 183 (184); F. Bucher, Design in Gothic Architecture - A Preliminary Assessment, The Journal of the Society of Architectural Historians 27 (1968), S. 49 ff.

22 Siehe Patterson, On the Margin (Fn. 6), S. 92; T. Burrows, Unmaking ,the Middle Ages“, Journal of Medieval History 7 (1981), S. 127.

23 D. R. Kelley, Civil Science in the Renaissance - Jurisprudence Italian Style, Historical Journal 22 (1979) S. 777 (778).

24 Zur Einführung etwa P. Stein, Roman Law in European History 38 ff., Cambridge University Press 1999; Wieacker, Privatrechtsgeschichte (Fn. 5), S. 45 ff.; S. Kuttner, The Revival of Jurisprudence, in: R. L. Benson/G. Constable (Hrsg.), Renaissance and Renewal in the Twelfth Century, Cambridge, Mass.: Harvard University Press 1982, S. 299 ff.; siehe auch M. Bellomo, Società e diritto nell'Italia medievale e moderna, Rom: Il Cigno 2002, S. $261 \mathrm{ff.}$

25 Locus classicus ist C. H. Haskins, The Renaissance of the Twelfth Century, Cambridge, Mass.: Harvard University Press 1955; aus der jüngeren Literatur vgl. R. N. Swanson, The Twelfth-Century Renaissance, Manchester University Press 1999; Kuttner, Revival (Fn. 24), S. 304 ff. Einen Überblick über die jüngste Entwicklung bietet L. Melve, „The Revolt of the Medievalists“ - Directions in Recent Research on the Twelfth-Century Renaissance, Journal of Medieval History 32 (2000) S. 231 (238 ff. und passim).

26 Haskins, Renaissance (Fn. 25), S. 62. Ein Beispiel für die Entwicklung städtischen Lebens bietet etwa J. H. Mundy, Urban Society and Culture - Toulouse and its Region, in: R. L. Benson/G. Constable (Hrsg.), Renaissance (Fn. 24).

27 Siehe Haskins, Renaissance (Fn. 25), S. 93, 127 ff.; Swanson, Twelfth-Century (Fn. 25), S. 41 ff. 
dem Studium der artes liberales, în diesem propaedeuticum erlernten Regeln statt. $^{28}$ Anders als in Paris gab es zwar in Bologna zunächst keine Artistenfakultät; ${ }^{29}$ gleichwohl konnte der akademische Rechtsunterricht gar keine andere Form annehmen als die von der scholastischen Methode vorgegebene. Die Scholastik war mit dem Konzept der Universität untrennbar verbunden, nachgerade synonym mit professionalisierter intellektueller Betätigung - war, wie Jakob H.J. Schneider formuliert hat „Wissenschaft in der Gestalt der Schule“.30

Die scholastische Denkweise erklärt sich daher insbesondere in ihrer institutionellen Verknüpfung mit und Bedingtheit durch die spezifischen Erscheinungsformen mittelalterlicher Schulerziehung. Diese Orientierung am Paradigma der „Schule“ erhellt die pädagogische Ausrichtung der scholastischen Denkweise, die - wenn man so will - als eine Frühform der Einheit von Forschung und Lehre - nicht nur den akademischen Unterricht charakterisiert, sondern schlichtweg jedwede Form wissenschaftlicher Tätigkeit beherrscht. Der innige Zusammenhang zeigt sich auch im rechtswissenschaftlichen Schrifttum an den typischen Formen wie der Glosse und der Quaestio. ${ }^{31}$ Die Glosse hat ihren Ursprung als Notiz der Texterläuterung im Unterricht; die Quaestio im ritualisierten gelehrten Disput. ${ }^{32}$

\section{Text, Autorität, ratio scripta}

Die scholastische pädagogische ${ }^{33}$ wie auch wissenschaftliche Ambition galt zunächst dem Verstehen und Erklären der auctoritates - Texte, die als Ausdruck alter und damit höherer Weisheit galten und denen es sich mithin mit intellektueller Demut zu nähern galt. ${ }^{34}$ Die Werke der auctores waren grundsätzlich weder zu kritisieren noch gar zu falsifizieren. Gleichwohl, selbst von diesem Ausgangspunkt äußerster Reverenz zielt intellektueller Ehrgeiz letztlich, und wenn auch nur ein wenig, höher - der Gelehrte, der auf den Schultern von Riesen steht, hat darum auch einen besseren Ausblick. ${ }^{35}$ Dieses Mehr war die Fähigkeit, die Quellen mit Hilfe der im trivium

28 G. Leff, The Trivium and the Three Philosophies in A History of the University in Europe, in: W. Rüegg (Hrsg.), Universities in the Middle Ages, Vol. I, Cambridge University Press 1992, S. 307 ff. (310); Swanson, Twelfth-Century (Fn. 25), S. 29 ff.

29 A.B. Cobban, The Medieval Universities, London: Methuen \& Co 1975, S. 61; J.H.J. Schneider, Wissenschaftseinteilung und institutionelle Folgen, in: M. Hoenen/J.H.J. Schneider (Hrsg.), Philosophy and Learning - Universities in the Middle Ages, Leiden: E.J. Brill 1995, S. 63 (67).

30 Schneider, Wissenschaftseinteilung (Fn. 29), S. 68.

31 H. Kantorowicz, The Quaestiones Disputatae of the Glossators, Tijdschrift voor Rechtsgeschiedenis 16 (1939), S. 1 ff.; A. Belloni, Le questioni civilistiche del secolo XII: da Bulgaro a Pillio da Medicina e Azzone, Frankfurt a.M. 1989, S. 3-65.

32 W.J. Hoye, Die mittelalterliche Methode der Quaestio, in: N. Herold/B. Kensmann/S. Mischer (Hrsg.), Philosophie - Studium, Text und Argument, Berlin et al. 1997, S. 155.

33 Zur „scholastischen Pädagogik“ siehe J. Verger, Patterns, in: Rüegg (Hrsg.), Universities (Fn. 28), S. $41 \mathrm{ff}$.

34 Wieacker, Privatrechtsgeschichte (Fn. 5), S. $54 \mathrm{ff}$.

35 J. Saresberiensis, Metalogicon, Lib. III, Cap. IV. 
angeeigneten ratio $^{36}$ zu erläutern, ihren Sinn zu erschließen und diese Erkenntnis an Schüler weiterzureichen.

Allein die übermäßig respektvolle, ja devote Haltung gegenüber den Autoritäten will uns fremd anmuten, scheint uns noch am ehesten nachvollziehbar für die Exegese religiöser Texte. ${ }^{37}$ Doch auch säkulare Texte, klassische Literatur etwa, ${ }^{38}$ wurden mit ähnlich quasi-religiösem Respekt behandelt. Die Digesten, komplexeste und reichhaltigste Sammlung juristischer römischer Quellentexte, galten als „ratio scripta“.${ }^{39}$ Deutlichster Ausdruck der intellektuellen Selbstbescheidung sind die Anfänge der zunächst rein erläuternden Glossenliteratur, die bereits in ihrem optischen Erscheinungsbild die Demutshaltung gegenüber dem autoritativen Text artikuliert. ${ }^{40}$ Mit zunehmendem Ehrgeiz und Selbstbewusstsein konnte sich die Textinterpretation nach und nach zu immer größerer intellektueller Freiheit aufschwingen, konnte, in den Worten von Knut Wolfgang Nörr, im „Verschnitt“ der Komponenten der mittelalterlichen Geistigkeit, ${ }^{41}$ die ratio und damit die Spielräume des Interpreten immer größeren Raum einnehmen - was sich auch in den Äußerungsformen der mittelalterlichen Rechtsgelehrten niederschlägt, der Glosse, die bald die Quellentext im Umfang weit übertrifft, Summen und letztlich Kommentare. Trotz des freieren Umgangs wird der Textbezug derweil nie gänzlich aufgegeben, und auch nicht die Grundannahme der Autoritativität des Textes.

Die Digesten, zur „ratio scripta“ stilisiert, mussten den mittelalterlichen Interpreten vor besondere Schwierigkeiten stellen, finden sich doch in der schieren Masse der Quellen stets dunkle Passagen und innere Widersprüche. Diese Widersprüche suchte die Rechtswissenschaft auszuräumen; durch Erläuterung und geschickte Auslegung zwischen den Quellen „concordantia“ zu erzeugen. Die wichtigste zu diesem Ende angewandte Technik war die der „disctinctio“, die in einem ersten synthetischen Schritt einen Begriff aus den Quellen induzierte, um dann von diesem Oberbegriff

36 Siehe K.W. Nörr, Von der Textrationalität zur Zweckrationalität - Das Beispiel des summarischen Prozesses, Zeitschrift der Savigny-Stiftung für Rechtsgeschichte Kan. Abt. 81 (1995), S. 1 (2); G. Otte, Dialektik und Jurisprudenz, Untersuchungen zur Methode der Glossatoren, Frankfurt am Main 1971. Otte vertritt den Standpunkt, die Glossatoren seien in ihrer Dialektik weit über den Standard des trivium hinausgegangen (S. 21). Berman, Law (Fn. 1), S. 143 ff., nennt Gratians Decretum als "[p]robably the most striking single example of the role of the scholastic dialectic in the formation of Western legal science".

37 Siehe etwa P. Goodridge, Historical Aspects of Legal Interpretation, Ind. L. J. 61 (1985-1986), S. 331 (335 ff.); C. Brown, Contrary Things - Exegesis, Dialectic, and the Poetics of Didacticism, Stanford University Press 1998, S. 15 ff.

38 Siehe S. Reynolds, Medieval Reading - Grammar, Rhetoric, and the Classical Text, Cambridge University Press 1996.

39 Wieacker, Privatrechtsgeschichte (Fn. 5), S. 55.

40 Goodridge, Historical Aspects (Fn. 37), S. 338 ff.; ibid. S. 35 f.

41 Nörr, Textrationalität (Fn. 36), S. 1. 
im Wege der Distinktion Unter- und Unter-Unterbegriffe abzuleiten. ${ }^{42}$ Zum Einsatz gelangt eine „rationale“ Methode, die ratio der Schulweisheit, ${ }^{43}$ um die Widerspruchslosigkeit, die Wahrheit eines aufgrund der Umstände seiner Entstehung, der Kompilation durch die oströmischen Juristen - fehlbar und aus Fleisch und Blut notwendig imperfekten Rechtstextes unter Beweis zu stellen. Dem modernen Menschen muss diese Mittel-Zweck-Relation als seltsam gebrochen und irrational erscheinen: Wir können, vom Standpunkt unserer Rationalitätsvorstellung, nicht begreifen, wie der mittelalterliche Jurist glauben konnte, im Corpus Iuris sei „die Vernunft selbst Wort geworden." 44

Dass es als problematisch empfunden wird, wenn sich Rechtstexte widersprechen, die als autoritativ und damit als normativ anerkannt werden, ist indes ohne weiteres einsichtig. Es handelt sich gewissermaßen um ein hermeneutisches Ewigkeitsproblem, das immer dann auftritt, wenn sich ein Interpret verbindlichen Texten gegenübersieht - „[d]ie Aufgabe des Verstehens und Auslegens besteht eben nur dort, wo etwas so gesetzt ist, dass es als das Gesetzte unaufhebbar und verbindlich ist." 45

Dem Interpreten moderner Gesetzestexte stellt sich strukturell dasselbe Problem. Fremd ist uns allein die Entscheidung hinsichtlich dessen, was als „gesetzt“ - als das „Gesetz" gelten kann. Bei der Auslegung des Corpus Iuris erscheint es intuitiv als „irrational“, dessen Historizität auszublenden und durch die Unfehlbarkeitsprojektion der "ratio scripta“ zu ersetzen. Die Verbindlichkeit des Gesetzes, dem wir durch Auslegung Sinn zu entnehmen versuchen, ist freilich ebenfalls nichts anderes als ein Axiom, dem es sich - da für die Zielvorstellung „Rechtsstaat“ unentbehrlich - zu unterwerfen gilt. Auch Gesetze sind von Menschenhand gemacht und von inneren Widersprüchen geplagt, und gleichwohl wird das transzendentale Postulat vom Gesetz, das klüger sei als seine Väter, wenn auch mitunter murrend, hingenommen. Noch deutlicher wird die Parallele im common law: Ein Rechts-,System“ aus disparaten Obergerichtsentscheidungen extrapolieren zu wollen heißt, diese als „gesetzt" hinzunehmen ${ }^{46}$ - trotz notwendig auftretender Widersprüche müssen die $\mathrm{Au}$ toritäten „reconciled“ - „versöhnt“ - werden. Die moderne „rationalisierende“47 Schule anglo-amerikanischer Rechtstheorie tut eben dies: Sie sanktioniert die Erfin-

42 Eine ausführliche Darstellung, einschließlich der historischen Wurzeln in der griechischen diairesis bietet C.H.F. Meyer, Die Distinktionstechnik in der Kanonistik des 12. Jahrhunderts 63 ff. (Leuven University Press 2000).

43 Siehe Otte, Dialektik (Fn.36).

44 Wieacker, Privatrechtsgeschichte (Fn. 5), S. 55.

45 H.-G. Gadamer, Wahrheit und Methode - Grundzüge einer philosophischen Hermeneutik, Tübingen, J.C.B. Mohr (Paul Siebeck) 1990, S. 335

46 Wie problematisch diese Annahme ist, zeigt A.W.B. Simpson, The Common Law and Legal Theory, in: ders. (Hrsg.), Legal Theory and Legal History - Essays on the Common Law, London: Hambledon Press 1987, S. 359 (366 ff.), was ihn zu der Frage führt: „Is the common law a fiction?“ (S. 370).

$47 \mathrm{Zu}$ einer Deutung dieser „rationalisierenden“ Rechtstheorie siehe R.M. Unger, What should Legal Analysis Become?, London: Verso 1996, S. 36 ff. 
dung von Prinzipien ex post, als deren Ausdruck sich Gerichtsentscheidungen dann rational erklären lassen. Auch hier muss stillschweigend eine höhere Vernunft am Werke mitgedacht werden, deren Ursprung unklar ist. Systeme, die mit dem Paradigma der Geltung operieren, kommen letztlich nicht ohne „Grundnorm“ aus, die stets ihrem Wesen nach axiomatisch und deren Begründung in sich selbst liegt.

\section{Schul-Unterricht}

Die besondere Art und Weise, das Wie, mit dem sich die Scholastiker der Harmonisierung des Quellenmaterials annahmen, erklärt sich wiederum - zumindest teilweise - aus dem Hintergrund des pädagogischen Naturells scholastischen Denkens. In seinem Essay „Gothic Architecture and Scholasticism" arbeitet der Kunsthistoriker Erwin Panofsky die Herstellung von „concordantia“ als eine der beiden wichtigsten Ambitionen scholastischer Methode heraus. ${ }^{48}$ Dieses „logische“ procedere war, im juristischen Kontext, als wichtige Methode der „wissenschaftlichen“ Rechtsfindung akzeptiert - wohlbekannt ist das Rechtssprichwort: „Bene judicat qui bene distinguit“. Die Distinktionstechnik erklärt sich jedoch wiederum in ihren erzieherischen Wurzeln: „Bene docet, qui bene distinguit“ - wer gut distinguiert, lehrt gut, hieß ein anderes Sprichwort. ${ }^{49}$ Auch die Schüler musste es in Verwirrung stürzen, wenn die Autoritäten sich widersprachen. Die Herstellung von Konkordanz durch Distinguieren und die Schaffung von Begriffskategorien etabliert zugleich ein taxonomisches Koordinatensystem, das es dem Juristen erleichtert, das sperrige Quellenmaterial, die unzähligen Einzelfälle, abstrahiert zu bündeln und damit erlernbar zu machen.

Auch dies ist uns nicht fremd. Die „Begriffsjurisprudenz“ mag als überwunden gelten; allein ohne eine ordnende (d.h. nicht als rechtsschöpfend verstandene) Begriffsbildung wäre dogmatisches Denken und auf dessen Vermittlung zielender Rechtsunterricht undenkbar. Im common law, insbesondere im Vereinigten Königreich und dem Commonwealth, erfreut sich derzeit das Projekt der „Kartierung“ - eben einer taxonomischen Erfassung - des Common Law zu erklärtermaßen pädagogischen Zwecken großer Beliebtheit. ${ }^{50}$ Eine taxonomische Ordnung oder „Karte“, in der jedem Konzept ein Platz zugewiesen ist, hilft zudem nicht nur, Recht dem Schüler verstehbar zu machen, sondern auch memorierbar. Die mittelalterliche Erinnerungskunst war weit gediehen ${ }^{51}$ und wusste um den Wert taxonomischer Ordnung für die

48 E. Panofsky, Gothic Architecture and Scholasticism, Archabby Publications 21 (2005), S. 64; in diesem Kontext bezieht sich auch Meyer, Distinktionstechnik (Fn. 42), S. 63 f., auf Panofskys Behandlung der concordantia.

49 Meyer, Distinktionstechnik (Fn. 42), S. 65.

50 Dazu zusammenfassend H. Dedek, Border Control - Some Comparative Remarks on the Cartography of Obligations, in: J. Neyers et. al. (Hrsg.), Exploring Contract Law, Oxford: Hart 2009, S. 25 ff.

51 Einen Überblick zur mittelalterlichen Mnemotechnik bieten M. Carruthers/J.M. Ziolkowski, The Medieval Craft of Memory, An Anthology of Texts and Pictures, University of Pennsylvania Press 2002. 
Mnemotechnik: ${ }^{52}$ Taxonomien sind visualisierbar, nicht nur in metaphorischer Form (wie ebenfalls als Memorierhilfe anempfohlen), ${ }^{53}$ sondern auch unmittelbar in Anschauungshilfen wie Tabellen und Diagrammen. Um distinctiones zu visualisieren, boten sich Baumdiagramme als geradezu „natural and fitting“ $\mathrm{an}^{54}$ - eines derjenigen pädagogischen Hilfsmittel, die sich noch heute in jedem Repetitorenskript finden.

Die Vergegenwärtigung der pädagogischen Anknüpfung hilft auch beim Verständnis der „manifestatio“ als der zweiten von Panofsky herausgearbeiteten Hauptambition scholastischer Vorgehensweise“. ${ }^{55}$ Manifestatio verlangt die Erläuterung des Argumentationsprozesses selbst und zeichnet daher mitverantwortlich für den vielkritisierten Formalismus scholastischer Literatur. Nach Panofsky bedeutet dies im einzelnen „1) totality (sufficient enumeration), 2) arrangement according to a system of homologous parts and subparts (sufficient articulation) and 3) distinctness and deductive cogency (sufficient interrelation). " 56 Panofsky erinnert zurecht daran, was für eine erhebliche Zivilisationserrungenschaft dieses - uns selbstverständlich scheinende - Bestehen auf stringenter Gliederung ist: Während klassische Literatur zumeist nur in „libri“ gegliedert ist, führt die Scholastik die Unterteilung von Texten in Abschnitte und Unterabschnitte ein, die den dem Text zugrundeliegenden Gedankengang offenlegt. ${ }^{57}$ Auch hier ist das Ziel, bei der Erläuterung der Quellen ein Höchstmaß an Klarheit zu erreichen und zugleich Rechenschaft über die eigene Vorgehensweise abzulegen.

Schulweisheit, so können wir zunächst festhalten, bildet spezifische Gesetzmäßigkeiten aus, gehorcht einer gleichsam über-kulturellen Rationalität - ein Phänomen, das der Soziologe Edgar Zilsel wie folgt umschrieben hat:

„Schoolteachers [...] take an interest in rational distinction and classifications. The old sentence, „,bene docet qui bene distinguit,” is as correct as it is sociologically significant. Schoolteaching, by its sociological conditions, produces a specific kind of rationality which appears in similar forms wherever old priests, entrusted with the task of instructing priest candidates, rationalize vague and

$52 \mathrm{Zu}$ dem Zusammenhang zwischen juristischer Methode und Mnemotechnik siehe C.H.F. Meyer, Spuren im Wald der Erinnerung - Zur Mnemotechnik in Theologie und Jurisprudenz des 12. Jahrhunderts, Recherches de Théologie et Philosophie Médiévales 67 (2000), S. 10.

53 F.A. Yates, The Art of Memory, University of Chicago Press 1966, S. $82 \mathrm{ff}$.

54 J.E. Murdoch, Album of Science, Antiquity and the Middle Ages, New York: Charles Scribner's Sons 1984, S. 38 ff.. Zu Visualisierung in wissenschaftlichem Schrifttum generell D. Topper, Towards an Epistemology of Scientific Illustration, in: B.S. Baigrie (Hrsg.), Picturing Knowledge, Historical and Philosophical Problems Concerning the Use of Art in Science, University of Toronto Press 1996, S. $215 \mathrm{ff}$.

55 Panofsky, Gothic Architecture (Fn. 48), S. 31.

56 Ibid.

57 Ibid. S. 32. 
contradictory mythological traditions of the past. Brabmans in India, Buddhist theologians in Japan, Arabic and Catholic medieval scholastics conform in their methods to an astonishing degree." 58

Den modernen law-schoolteacher, den Rechts-Lehrer, können wir dieser Aufzählung getrost hinzufügen: Auch hier werden von den Lehrern Adepten initiiert, in die Kunst eingeführt, die autoritativen Texte richtig zu verstehen und auszulegen - und dabei letztlich, wie wir gesehen haben, durchaus das ein oder andere „mythische“59 Element zu rationalisieren.

\section{Scholastik als Stil und „mentaler Habitus“}

Gewisse Axiome werden auch von dem modernen Juristen traditionell nicht hinterfragt. Die Traditionen nehmen es uns ab, über eine Vielzahl weiterer Dinge zu reflektieren: Komplexität wird reduziert, indem zahlreiche Vorgänge, die traditionellen Regeln gehorchen, nicht stets neu überdacht werden müssen. Die Sozialisierung in derartige Traditionen - zunächst als "Jurist“ im Allgemeinen, aber sodann auch in die jeweiligen Institutionentraditionen als Richter, Verwaltungsjurist, Notar etc. führt dazu, dass man Dinge in einer bestimmten Art und Weise tut, ohne darauf Gedanken zu verwenden. Die Rechtsvergleichung zeigt, wie wesentlich solche Unterschiede sein können, die sich in der verschiedensten Weise zeigen können: Wie Urteile verfasst, wie Schriftsätze gestaltet, wissenschaftliche Aufsätze geschrieben werden - Äußerlichkeiten mitunter, die in der Summe jedoch darüber Aufschluss bieten, wie insgesamt über „Recht“ gedacht und mit „Recht“ umgegangen wird. Nicht jeder Aspekt traditionsgebundenen Handelns ist rational erklärbar. So sind manche der Charakterzüge scholastischen Argumentierens vor dem Hintergrund des Schul-Paradigmas, der institutionellen Anknüpfung am Unterricht besser verständlich. Die besondere Ziselierung des Arguments indes, die Lust an der Subtilität der Distinktionen lassen sich mit utilitaristischen Erklärungen jedoch schwerlich fassen. Hier scheint etwas anderes am Werke als die Umsetzung einer als rational richtig erkannten Methodologie.

\section{I. „Rechtsästhetik“?}

Wie diesen Faktor zumeist unreflektierten und daher umso schwerer fassbaren Faktor erklären? Die Tradition der Rechtsgelehrsamkeit im Westen ist selbst eine Tradition „,wissenschaftlicher Rationalität“ ${ }^{“ 0}$, die daher traditionell nur wenig Toleranz

58 E. Zilsel, The Sociological Roots of Science, The American Journal of Sociology 47 (1942), S. 544 (548).

59 D.D. Barnhizer, Hypocrisy \& Myth - The Hidden Order of the Rule of Law, Lake Mary: Vandeplas 2009, S. 185 ff. zu dem Thema, dass auch Rechtsstaatlichkeit (rule of law) ein Ideal umschreibt, an das man glauben muss und auf das kollektiv hingearbeitet werden muss, um zumindest einen realen Näherungszustand zu erreichen.

60 H.P. Glenn, Legal Traditions (Fn. 10), S. 19. 
aufbringt, „irrationalen“ Faktoren Bedeutung einzuräumen. Während des letzten Jahrhunderts haben es Rechtsvergleicher unternommen, die Unterschiede zwischen Rechtssystemen, -kreisen oder -kulturen einzufangen, indem sie die Summe derer charakteristischen Eigentümlichkeiten als „Stil“ zu umschrieben. ${ }^{61}$ Der Komparatist René David ging gar so weit, das kontinentale Zivilrecht als solches einen „Stil“ zu nennen. ${ }^{62}$ Die herkömmliche Rechtstheorie, hauptsächlich mit der Beschreibung Rechts als normativem Regelsystem befasst, ${ }^{63}$ hat kaum Energie darauf verwendet, dieses Phänomen theoretisch zu umschreiben. Das theoretische Schrifttum, das sich nicht an jenem normzentrierten Diskurs beteiligt, ist, mit gewisser Berechtigung, als „apocryphal jurisprudence“ 64 bezeichnet worden. Teil dieses weniger sichtbaren Diskurses ist auch das (vielfach außerhalb der Rechtsfakultäten geführte) Gespräch um „legal aesthetics“ - Rechtsästhetik.

Schon die Wortschöpfung mag paradox anmuten. Um „ästhetische“ Metaphern, gerade aus dem Bereich der Architektur, waren Rechtsgelehrte zwar nie verlegen. ${ }^{65}$ Wissenschaftliche Befassung mit dem Thema schien indes lange untunlich: Der Vernunft (und dem Recht als deren höchstem Ausdruck) einerseits und der Ästhetik andererseits hat unsere Aufklärungstradition gänzlich unverbundene Bereiche zugewiesen. Als eine der Ausnahmen verdient Erwähnung etwa die 1946 erschienene Schrift „Vom Stil des Rechts“ des Staats- und Völkerrechtslehrers Heinrich Triepel. ${ }^{66}$ Wenn der Autor eines Nachrufs versichert, dass Triepel, seiner „Vertiefung in jene Randgebiete“ zum Trotze, „,durch und durch Jurist“ geblieben sei, ${ }^{67}$ so offenbart das den Generalverdacht unjuristischer Umtriebe, den die Befassung mit dem Thema hervorruft. Die Masse des Schrifttums, die sich diesem Themenfeld widmet, stammt denn auch aus der jüngeren Vergangenheit und zumeist aus Nordamerika. ${ }^{68}$ Der Vorwurf der mangelnden Seriosität wird hierzulande noch immer artikuliert. ${ }^{69}$

61 Vgl. etwa J.H. Merryman, The Italian Style I - Doctrine, 18 Stan. L. Rev. 39 (1965), S. 39-65; M. Van Hoecke/M. Warrington, Legal Cultures, Legal Paradigms and Legal Doctrine - Towards a New Model for Comparative Law, I.C.L.Q. 47 No. 3 (1998) S. 495.

62 Siehe zu diesem Zitat N. Kasirer, in: ders. (Hrsg.), Le droit civil, avant tout un style?, Montréal: Les Éditions Thémis 2003, S. IX ff.

63 Siehe D. Manderson, Apocryphal Jurisprudence, Studies in Law, Politics and Society 23 (2001) S. 81; ders., Songs without Music - Aesthetic Dimensions of Law and Justice, University of California Press 2000.

64 Manderson, Aprocryphal, (Fn. 63), S. 84.

65 Siehe etwa R. von Jhering, Geist des römischen Rechts, Bd. 2.1, 3. Aufl., Leipzig 1874, S. 33 f.

66 H. Triepel, Vom Stil des Rechts, Beiträge zu einer Ästhetik des Rechts (1947), Nachdruck Berlin 2007.

67 C. Bilfinger, In memoriam Heinrich Triepel 1868-1946, Zeitschrift für ausländisches öffentliches Recht und Völkerrecht 13 (1951-1952) S. 1 (2).

68 Eine schöne Einführung und einen umfassenden Überblick in deutscher Sprache geben A. von Arnauld/ W. Durner, Heinrich Triepel und die Ästhetik des Rechts, in: Triepel, Stil (Fn. 66), S. V (Xff.).

69 Vgl. etwa die von großer Indigniertheit zeugende „Marginalie“ von T. Ditt, Rumpelstilzchen, Rechtsgeschichte 12 (2008), S. 237 f. 
Das Reizwort „Ästhetik“ darf aber in unserem Kontext nicht zu falschen Schlüssen verleiten; „Ästhetik“ soll sich nicht auf die Bewertung und das Erleben des „Schönen " beziehen und beschränken. Es geht nicht darum, eine Brücke zur Kunst zu schlagen in der Weise, dass Recht in Kunstwerken nachgespürt oder Kunstwerke als Metaphern genutzt werden (wie etwa teilweise in Schriften der "law and literature" Bewegung - Josef Kohler war ein Vorreiter oder, wenn man so will, ein früher Vertreter dieser Richtung) oder, umgekehrt, gar darum Recht als Kunst zu stilisieren ( "law as art“). Dergestalt allein assoziiert mit „Kunst“ und „Schönheit“ ist der Vorbehalt gegen die Invokation der Kategorie „Ästhetik“ nachvollziehbar, scheint sie doch dem Ernst der auf dem Spiel stehenden Interessen nicht gemäß, ihnen gar Hohn zu sprechen: "[T]hat law is an aesthetic enterprise“, schreibt der Rechtstheoretiker Pierre Schlag, „can easily be seen as cavalier, ethically obtuse, even cruel. “70 Ästhetisches Erleben jedoch ist allumfassend und eben nicht auf „Kunst“ beschränkt. ${ }^{71}$ „Ästhetik“ scheint Schlag daher der geeignete Begriff, um Eigenarten und Manierismen einer Rechtstradition zu umschreiben, die von den Traditionsteilnehmern nicht reflektiert werden, die nicht instrumentell oder funktionell erklärbar sind: „A legal aesthetic is something that a legal professional both undergoes and enacts, most often automatically, without thinking. " 72 Eine derart verstandene Rechtsästhethik hat mit Kunst zunächst nichts zu tun, äußert sich aber in Phänomenen wie dem „Stil“ einer bestimmten Rechtskultur.

\section{II. (Rechts-)Ästhetik im Mittelalter}

\section{Der ,italienische Stil“}

In der rechtsgeschichtlichen Forschung wird durchaus auf Phänomene wie den „Stil“ rekurriert, um die charakteristischen Merkmale von Epochen oder geistigen Strömungen zu erfassen; offensichtlichstes Beispiel ist das der „eleganten Jurisprudenz“, eine Bezeichnung, die in ihrer Verdichtung für das ganze Programm des methodisch wie stilistisch avancierten Humanismus steht. ${ }^{73}$ Das positiv konnotierte Epithet „elegant" diente just dazu, den Unterschied zwischen einem neuen und einem alten Stil $\mathrm{zu}$ umschreiben, eben dem scholastischen mos italicus. ${ }^{74}$ Auch die Bezeichnung „mos“, was man sowohl als Brauch und Sitte, aber auch als „Stil“ übersetzen

70 P. Schlag, The Aesthetics of American Law, Harv. L. Rev. 115 (2001-2002) S. 1047 (1050).

71 Manderson, Songs, (Fn. 63), S. 27-43.

72 Schlag, Aesthetics (Fn. 70), S. 1053.

73 Siehe etwa G.C.J.J. van den Bergh, Die holländische elegante Schule, Ein Beitrag zur Geschichte von Humanismus und Rechtswissenschaft in den Niederlanden 1500-1800, Frankfurt am Main 2002.

74 D.R. Kelley, Review - C.G.J.J. Van den Bergh, The Life and Work of Gerard Noodt (1647-1725) Dutch Legal Scholarship between Humanism and Enlightenment, Journal of Modern History 62 (1990), S. 643. 
kann, ${ }^{75}$ deutet darauf hin, dass hier nicht nur eine Methodologie durch eine andere ersetzt wird. Donald R. Kelley etwa verweist auf humanistische Polemiken, in denen die Proponenten des mos italicus als „Barbaren“ verspottet werden, frei von Geschichtskenntnis und ohne philologischen Geschmack. ${ }^{76} \mathrm{Um}$ sich von den scholastischen Gelehrten „alter Schule“ abzusetzen, hoben die Humanisten ihre Kultiviertheit und ihren gediegeneren Stil hervor - was uns im Gegenschluss genauso zeigt, dass die scholastische Wissenschaft von einer typischen Erscheinungsweise, einem Stil, gekennzeichnet war, von dem uns der Spiegel humanistischer Polemik nur ein allzu unvorteilhaft verzerrtes Abbild liefert.

\section{Scholastische „Ästhetik“?}

Der Versuch, verstehen zu wollen, welchen un- oder vorbewussten Mustern das Verständnis und die Praxis der scholastischen Rechtsgelehrten von wissenschaftlicher Textproduktion gehorchte, begegnet freilich nicht unerheblichen, in der „Natur“ des Untersuchungsgegenstandes begründeten Schwierigkeiten. Von dem überlieferten juristischen Schrifttum ist eine Reflektion über derartige - eben unausgesprochene, wahrscheinlich uneingestandene - Mechanismen, über solche „Rechts-Ästhetik“ kaum zu erwarten. Wenn wir unsere Suche auf eine scholastische „Ästhetik“ auch außerhalb der Rechtswissenschaft erweitern und auf andere Literaturen der Epoche blicken, so müssen wir wiederum auf Hut sein, nicht unseren modernen Erwartungshorizont an „Ästhetik“ anzulegen.

Der Begriff der „Ästhetik“ selbst, „Ästhetik“ als akademische „Disziplin“, die vornehmlich die Wahrnehmung von „Kunst“ im Sinne der „beaux arts“ zum Gegenstand hat und sich als Seitenstück zur Theorie der rationalen Erkenntnis entwickelt, ist ein Produkt des 18. Jahrhunderts, wie auch die Gleichsetzung von Kunst - ars mit den „schönen “ Künsten. ${ }^{77}$ Unter Kunsthistorikern und -philosophen ist daher die Frage diskutiert worden, inwieweit der Terminus „Ästhetik“ überhaupt Anwendung finden kann auf jenen mittelalterlichen Diskurs, ${ }^{78}$ der sich inhaltlich mit dem der modernen „Ästhetik“ überlappt. ${ }^{79}$ Dieser Frage müssen wir jedoch nicht nach-

75 So schlägt etwa das Oxford Latin Dictionary, Oxford 1983, s.v. „mos”, die Übersetzung „style“ vor; Langenscheidts Großwörterbuch Lateinisch: Lateinisch-Deutsch, München 2001, s.v. „mos”, kennt neben „Sitte“, „Gewohnheit“, „Brauch“ etc. zudem auch „Art und Weise“ und „Mode“.

76 D.R. Kelley, Civil Science in the Renaissance - Jurisprudence Italian Style, Historical Journal 22 (1979) S. 777 (778).

77 P.O. Kristeller, The Modern System of the Arts - A Study in the History of Aesthetics Part I, Journal of the History of Ideas 12 (1951), S. $496 \mathrm{f}$. und passim.

78 J.A. Aertsen, Beauty - Medieval Concepts, in: M. Kelly (Hrsg.), Encyclopedia of Aesthetics, Vol. 1, Oxford University Press 1998, S. 251 ff.; J. Margolis, Medieval Aesthetics, in: B.N. Gaut/D.M. Lopes (Hrsg.), The Routledge Companion to Aesthetics, London/New York: Routledge 2005, S. 29 (, contrived“, aber letztlich „unavoidable“). Zu Benedetto Croce (ästhetisches Empfinden als „lyrical intuition of feeling“) siehe U. Eco, The Aesthetics of Thomas Aquinas (übers. von H. Bredin), Cambridge, Mass.: Harvard University Press 1988, S. 1 ff.

79 W. Tatarkiewicz, History of Aesthetics, in: C. Barrett (Hrsg.), Medieval Aesthetics, Vol. II, Den Haag: Mouton 1970, S. 287 ff. 
gehen; für uns ist gerade hilfreich, dass es den mittelalterlichen Überlegungen zu dem, was schön und darum auch gut ist, die Fixierung auf den von der Vernunft abgetrennten Bereich der „beaux arts" noch fehlt; dass sie sich vielmehr auf jeden Lebensbereich und damit auch auf intellektuelles Schaffen beziehen. Die scholastischen Reflektionen über Schönheit, Harmonie und Konkordanz betreffen uns, weil sie nicht auf die vom idealisierten Künstler-Genie produzierte „Kunst“ beschränkt ist.

Das, was in der Literatur als „ästhetische Theorien“ der Scholastik bezeichnet worden ist, ${ }^{80}$ befasst sich mit „Kunst“ im Sinne von „ars“ in der Wortbedeutung, die uns etwa bei den "artes liberales“, den freien Künsten, bereits begegnet ist. „Ars“ umfasste nahezu jede Form von Schaffenstätigkeit, sei es im physischen oder im intellektuellen Sinne. ${ }^{81}$ Dergestalt umschrieb „ars“ etwa die Handwerkskunst ebenso wie die „freien Künste“; in den Worten des Kunsthistorikers Paul Kristellers: "shoemaking, cooking and juggling, grammar and arithmetic [were] no less and in no other sense artes than painting and sculpture, poetry and music". ${ }^{82}$ "Ars" bedeutete schlicht: zu wissen wie man etwas zusammenfügt, herstellt, macht..$^{83}$ Die Theorien über die „Schönheit“, die ebenfalls noch nicht auf ein rein äußerliches Erscheinungsbild reduziert ist, betreffen daher jeden Schaffensprozess, waren, wie Kunsthistoriker De Bruyne feststellt, „as valid for the doctor, laborer, shoemaker as it is for the painter and sculptor. " 84 Die mangelnde Ausdifferenzierung und fehlende wechselseitige Abschottung von Lebensbereichen ist charakteristisch für die Epoche - man rufe sich nur in Erinnerung, dass die auch die Institutionalisierung von Wissen in der frühen Universität in ihrem Selbstverständnis dem Vorbild der Zünfte und Gilden („arti“) folgte. ${ }^{85}$

Lediglich dem gängigen modernen Verständnis von „Ästhetik“ - das wir in unserem obigen Definitionsversuch bereits zu meiden versuchten - muss es daher eigenartig erscheinen, wenn Proportion und Harmonie als Kategorien des „Schönen“ bei den Scholastikern, ausdrücklich auch auf rationalen Diskurs angewendet werden; so kommentiert etwa Umberto Eco eine derartige Äußerung bei Thomas von Aquin mit den Worten, dies möge der modernen Anschauung zwar prima vista „pervers“ erscheinen, es sei allerdings durchaus eine Rückkehr zu der Auffassung zu verzeichnen,

80 U. Eco, Art and Beauty in the Middle Ages, (übers. von H. Bredin), Yale University Press 1986, S. 21.

81 W. Tatarkiewicz, Did Aesthetics Progress?, Philosophy and Phenomenological Research 31 (1970), S. 46 (52).

82 Kristeller, System (Fn. 77), S. 508 ff.

83 Eco, Aesthetics (Fn. 78), S. 164.

84 E. De Bruyne, The Esthetics of the Middle Ages (übers. von E.B. Hennessy), New York: Frederick Ungar 1969, S. $180 \mathrm{ff}$.

85 J.W. Baldwin, The Scholastic Culture of the Middle Ages 1000 - 1300, Lexington: D.C. Heath and Company 1971, S. $40 \mathrm{f}$. 
dass auch Rationales und Logisches eine ästhetische Dimension aufweise. ${ }^{86}$ Es besteht kein Anlass, die Produktion (rechts-)gelehrter Schriften von dieser mittelalterlichen „ästhetischen“ Anschauung ausgeschlossen zu halten.

\section{Erwin Panofsky: Scholastik als "mentaler Habitus“}

Die These, dass sich umgekehrt der scholastische modus operandi nicht nur in der Behandlung intellektueller Fragestellungen, sondern auch in anderen Schaffensformen äußert, hat vor allem Erwin Panofsky in seiner Vorlesung über „Gothic Architecture and Scholasticism" vertreten. Die These besagt, auf das wesentlichste verkürzt, gotische Architektur sei in Stein gefügte Scholastik, ${ }^{87}$ wobei diese nicht materiell als Kanon philosophischer oder theologischer Ansichten aufgefasst wird, sondern als „mental habit“, als „mentaler Habitus“. Derart umfassend gedacht, ist die Scholastik ebenso wissenschaftliche Methode wie „Stil“, „ästhetisches“ Prinzip. Panofskys Argument können wir für unsere Zwecke wenden: Für Panofsky ist die Symmetrie des Rippengewölbes ${ }^{88}$ oder der Fensterrosen ${ }^{89}$ mit ihren zahlreichen Unterteilungen in Stein gefügter scholastischer Rationalismus; die steingewordene Verkörperung dieser Prinzipien zeigt uns derweil unverkennbar deren „ästhetische“ Dimensionen auf. Einem ganz ähnlichen Gedankengang folgend wie Panofsky schreibt der Mediävist John W. Baldwin, dass die Schulkultur in der Weise auf die Architektur wirkte, dass Stilelemente wie eben die Fensterrose konstruiert wurden „according to the yeses and noes of the Sic et Non of Abelard or a thirteenth century disputation". Umgekehrt können wir daran ablesen, dass ein scholastischer Text, eine scholastische gelehrte Disputation so etwas wie einen Rhythmus, ein Metrum hat.

In der Visualisierung der scholastischen Prinzipien, der „visuellen Logik“ (Panofsky) der Kathedrale verdeutlicht sich, wie scholastischer Rationalismus nicht nur funk-

$86 E c o$, Art (Fn. 80), S. 77. Die Mathematik böte sich hier als Beispiel an, der es auch um die „Eleganz“ eines Beweises zu tun ist.

87 Zu den Verbindungslinien zwischen Scholastik und gotischer Architektur siehe etwa C.M. Radding/ W.W. Clark, Medieval Architecture, Medieval Learning - Builders and Masters in the Age of Romanesque and Gothic, Yale University Press 1992, S. 3 ff., 81 ff. und passim. Vgl. kritisch zu Panofsky: P. Kidson, Panofsky, Suger and St Denis, Journal of the Warburg and Courtauld Institutes 50 (1987), S. 1 ff.; S. Croddy, Gothic Architecture and Scholastic Philosophy, British Journal of Aesthetics 39 (1999), S. 263; E. Inglis, Gothic Architecture and a Scholastic - Jean de Jandun's Tractatus de laudibus Parisius, Gesta 42 (2003), S. 63 (73 ff.). Eine pragmatischere Sichtweise vertritt P. Frankl, Gothic Architecture (überarb. von P. Crossley), Yale University Press 2000, S. 295); indes: „[n]o theologian can have introduced the rib. "Siehe zuletzt M.T. Davis, "Sic et Non“ - Recent Trends in the Study of Gothic Ecclesiastical Architecture, The Journal of the Society of Architectural Historians 58 (1999) S. $414 \mathrm{ff}$.

88 Beispiele geben W. Taylor/R. Mark, The Technology of Transition - Sexpartite to Quadripartite Vaulting in High Gothic Architecture, The Art Bulletin 64 (1982), S. 579 (580).

89 Siehe etwa F. Bucher, Design in Gothic Architecture - A Preliminary Assessment, The Journal of the Society of Architectural Historians 27 (1968), S. 49 (52 f.) 
tionalen - hier etwa: statischen - Zwecken dient, sondern eben auch Selbstzweck ist. ${ }^{90}$ Der Architekturhistoriker Otto von Simson hat den Zusammenhang zwischen Funktion und Form in der Gotik dergestalt beschrieben, dass, anders als in der Romanik, das ästhetische System des gotischen Gebäudes durch seine statisch maßgeblichen Elemente wie Rippengewölbe und Strebebögen bestimmt ist. ${ }^{91}$ Dies führt uns zurück zu dem Postulat der „manifestatio“: 92 In der scholastischen Produktion wissenschaftlicher Texte sind Komposition, Struktur und Organisation als "tragende“ Elemente Mittel zum Zweck. Sie treten jedoch zugleich auch selbst in den Vordergrund und dominieren die Ästhetik des Texts.

Von besonderem Interesse ist indes, auf welche Weise Panofsky den Bezug zwischen akademischem Scholastizismus und dessen Wirken in der Architektur herstellt. Bei dem von ihm beschriebenen Phänomen handele es sich nicht um bloße Koinzidenz oder eine „Parallele“; vielmehr bestehe eine „genuine cause-and-effect-relation" 93 . Bedingt sei diese Kausalität durch die Natur der Scholastik als umfassendem „mental habit“, als "principle that regulates the act“ ${ }^{94}$ Die Erklärung Panofskys dafür, dass auch die mittelalterlichen Baumeister dieser Denkweise ausgesetzt waren, um sie anschließend selbst zu übernehmen, ist wegen der schwachen historischen Beweislage vielfach kritisiert worden. ${ }^{95}$ Die Frage nach den Kanälen der Einflussnahme müssen wir freilich gar nicht stellen, die wir uns mit scholastischen Gelehrten selbst befassen: mit Gelehrten, deren Lebenswelt die Schule war. Hier hilft uns Panofsky zu verstehen, dass die Scholastik weit mehr war als eine wissenschaftliche Methode, sondern vielmehr etwas weit umfassenderes, ein „mental habit“, der, in Fleisch und Blut übergegangen, Bewusstsein wie Unterbewusstsein bestimmte. Bezeichnenderweise hat Pierre Bourdieu auf Grundlage dieser Beschreibung Panofskys seine Theorie vom "Habitus“ entwickelt, ${ }^{96}$ der in der Sozialisierung gerade durch Ausbildungsinstitutionen wurzelt und als „strukturierende Struktur" ${ }^{\text {“97 }}$ bewusste wie unbewusste, ra-

90 O.G. von Simson, The Gothic Cathedral - Design and Meaning, The Journal of the Society of Architectural Historians 11 (1952), S. 6 (passim); M. Gelernter, Sources of Medieval Form - A Critical History of Western Design Theory, Manchester University Press 1999, S. 75 f. Zu der spirituellen und mystischen Symbolik siehe auch P. Fingesten, Topographical and Anatomical Aspects of the Gothic Cathedral, The Journal of Aesthetics and Art Criticism 20 (1961), S. 3 (passim).

91 O. von Simson, The Gothic Cathedral, 3. Aufl., Princeton University Press 1988, S. 5 f.

92 Siehe oben zu Fn. 55.

93 Panofsky, Gothic Architecture (Fn. 48), S. 20.

94 Ibid. S. 21.

95 Vgl. R. Branner, A Note on Gothic Architects and Scholars, The Burlington Magazine 99 (1957) S. 372: „But the factual evidence of contact between these men is woefully slim and comes only from the thirteenth century (...)".

96 P. Bourdieu, Intellectual Field and creative project, Social Science Information 8 (1969), S. 89 (116-118).

97 P. Bourdieu, Outline of a Theory of Practice (übers. von R. Nice), Cambridge University Press 1977, S.72. 
tionalisierbare und nicht rationalisierbare Handlungsmuster vorgibt. Die Scholastik ist das Paradigma einer solchen „strukturierenden Struktur“.

\section{Scholastische „Ästhetik“: Harmonie und Ordnung}

Wenn wir nun endlich scholastische - im dargelegten Sinne - „ästhetische“ Literatur näher betrachten, so stellt sich der Eindruck ein, dass scholastische Philosophie postuliert, was die scholastische Methode praktiziert: Scholastische „Ästhetik“ kreist um Ordnung, Harmonie, Proportion, Symmetrie, Kongruenz, Konsonanz, concordia, concordantia. Eines der sich am deutlichsten abzeichnenden Leitmotive, das die - im einzelnen erwartungsgemäß scholastisch-intrikaten - Theorien gemein haben ist, was Umberto Eco ein „quantitatives“ Verständnis des Schönen genannt hat, dessen Ursprünge sich bis in die griechische Antike, ${ }^{98}$ etwa zu Pythagoras und Polykleitos, ${ }^{99}$ zurückverfolgen lassen. Hiernach ergibt sich die Schönheit des Ganzen stets aus der harmonischen Proportioniertheit seiner Bestandteile. ${ }^{100}$ Dieser Ansatz, tradiert durch Augustinus und Boethius, ist Ausgangspunkt der Scholastiker und findet sich bei fast allen Autoren, Grosseteste, Bonaventura, Albertus Magnus, Thomas von Aquin und sogar Wilhelm von Ockham. ${ }^{101}$

Der Schlüsselbegriff der consonantia entstammt der Musiktheorie, welche als eine der freien Künste dem quadrivium angehörte. Es zeigt sich wieder die umfassende Wirkweise der Erziehung; Jan A. Aertsen hat festgestellt: „musical conception, which regards proportion and harmony as essential to beauty, [...] also influenced the Middle Ages through education in the liberal arts. "102

Ein weiteres, häufig anzutreffendes Thema ist das des „splendor formae“, des Glanzes oder der Pracht der „Form“. Diese mache das Schöne aus, wie es etwa bei Albertus Magnus und Thomas heißt: „ratio pulchri in universali consistit in resplendentia formae super partes materiae proportionatas, vel super diversas vires vel actiones“, 103 schreibt Albertus in seinem "Opusculum de pulchro et bono“. Die Vermutung läge nahe, in dieser Idealisierung der „Form“ den Ursprung der sprichwörtlichen Lust am Formalismus der Scholastiker zu suchen. In der Tat liegen die Dinge viel schwieriger: „forma“ wird von manchen Autoren im Sinne des griechischen morphê verwandt, was in der Tat das äußerliche Erscheinungsbild meint. ${ }^{104}$ Insbesondere als Teil der Wendung „splendor formae“ oder „resplendentia formae“

98 De Bruyne, Esthetics (Fn.84), S. 5 ff.; Eco, Art (Fn. 80), S. 28.

99 Siehe E. Panofsky, Meaning in the Visual Arts, University of Chicago Press 1982, S. 63-68.

100 Panofsky, Gothic Architecture (Fn. 48), S. 64.

101 Tatarkiewicz, Aesthetics (Fn. 81), S. 50; ausführlicher ders., History (Fn. 79), S. 213 ff. Zum topos der „Proportion“ in Wissenschaft und Philosophie siehe R. Padovan, Proportion, London: E \& FN Spon 1999, S. 116 ff. (Aristoteles), 137 ff. (Pythagoras), 156 ff. (Gotik).

102 Aertsen, Beauty (Fn. 78), S. 250.

103 Zitiert nach Tatarkiewicz, History (Fn. 79), S. 243.

104 Eco, Art (Fn. 80), S. 77. 
nimmt „forma“ jedoch eine andere Bedeutung an, die des „Wesens“ im Sinne von eidos. ${ }^{105}$ Diesem, in der Tradition der Aristotelischen Substanzlehre stehenden Ansatz zufolge setzt sich ein Einzelding jeweils aus „Materie“ und „Form“ (Wesen, eidos) zusammen. Ohne hier den „Hylemorphismus“106 des Albert und Thomas im Detail untersuchen zu können, zeigt sich doch, dass die Definition des Schönen im Sinne „splendor formae“ eben nicht eine "formale“ Definition ist, sondern just das Gegenteil. ${ }^{107}$ In Thomas' „Ästhetik“108 erlangt die derart verstandene Form indes ihren splendor mithilfe dreier konstitutiver Kriterien: Proportion oder „Maßverhältnis“ (proportio, consonantia, harmonia), Klarheit (claritas) und Ganzheit bzw. Vollendung (integritas, perfectio). Dies sind wiederum Formalkriterien, ${ }^{109}$ die sich auf Struktur, Anordnung und Organisation beziehen. Formaler Harmonie und Proportion lassen das innere Wesen aufscheinen, sie erhellen die „Form“ im Sinne des eidos, indem sie sie begreifbar machen. ${ }^{110}$

Von hier ist es nur ein kleiner Schritt zur Eluzidation eines Gedankens, einer Idee durch entsprechende Darstellung. Die Ideale von Proportion, Konsonanz etc., die alle scholastischen Schriften zu diesem Themenbereich durchziehen, beanspruchen allgemeine Geltung, finden nicht nur Anwendung auf physische Erscheinung, sondern auch auf „wohlproportionierte“ Gedanken. ${ }^{111}$ Konsonanz ist hierbei das Kriterium, dem unser Hauptaugenmerk zu gelten haben wird, wenn wir nun einen Blick darauf werfen, wie Widersprüche, „Dissonanzen“ zwischen autoritativen Quellen römischen Rechts aufgelöst wurden.

\section{Scholastik am Werk: Die Interessendoktrin}

Die bekannte Lehre vom Interesse erscheint als besonders geeignetes Beispiel für Methode und Stil einer juristischen Argumentationsweise, die dem scholastischen „Habitus“ entspringt. Der mittelalterliche Jurist war hier mit einer komplexen Quel-

105 Zur Aristotelischen Substanzlehre und der Beziehung zwischen „Materie“ und „Form“ siehe etwa K. Fine, Aristotle on Matter, Mind 101 (1992), S. 35 ff.

106 C.M. Cullen, Scholastic Hylomorphism and Western Art - From the Gothic to the Baroque, in: A. Ramos (Hrsg.), Beauty, Art and the Polis, The Catholic University of America Press 2000, S. 45 ff.

107 Tatarkiewicz, History (Fn. 79), S. 240.

108 Die Theorien des Thomas sind wahrscheinlich die am gründlichsten erforschten Aspekte mittelalterlicher „Ästhetik“: monographisch Eco, Aesthetics (Fn. 78); zuletzt K.E. O`Reilly, Aesthetic Perception, A Thomistic Perspective, Dublin: Four Courts Press 2007; zur Einführung Margolis, Aesthetics (Fn. 78), S. 33 ff.; A. Speer, Aquinas, Thomas, in: Kelly (Hrsg.), Encyclopedia (Fn. 78), S. 76. Vgl. auch C.S. Steinberg, The Aesthetic Theory of Thomas Aquinas, The Philosophical Review 50 (1941), S. 483 ff.; C. Barrett, The Aesthetics of St. Thomas Re-Examined, Philosophical Studies 12 (1963), S. 107.

109 O`Reilly, Perception (Fn. 108), S. 18. Siehe auch J. Kreuzer, Kunstphilosophie im Mittelalter. Von Augustinus (354-430) bis Nikolaus von Kues (1401-1464), in: S. Majetschak (Hrsg.), Klassiker der Kunstphilosophie - von Platon bis Lyotard, München 2005, S. 50: “...Kriterien der Bestimmung des Schönen, die es von seiner ,formal-objektiven' Seite her denken...".

110 Steinberg, Theory (Fn. 108), S. 495 f.

111 O'Reilly, Perception (Fn. 108), S. 20. 
lenlage konfrontiert, die es erschwerte, eine kohärente, lehrbare Theorie zu entwickeln und Konkordanz zwischen den Quellen herzustellen.

\section{Begriffsbildung und Distinktion - und ein „neuzeitlicher" Exzess}

Im klassischen römischen Formularprozess ${ }^{112}$ konnte in aller Regel das Urteil nur auf die Verurteilung zur Zahlung einer Geldsumme lauten. ${ }^{113}$ Die Höhe dieser Geldkondemnation wurde durch eine Schätzung (litis aestimatio) ermittelt, deren Maßstäbe an der jeweils verwendeten Klageformel ausgerichtet waren. War die Klagformel unbestimmt - etwa bei einer Verurteilung zur Leistung von Schadensersatz wegen eines Sachmangels ${ }^{114}$ oder grundloser Nichterfüllung des Kaufvertrages ${ }^{115}$ - auf eine Verurteilung „quidquid (...) dare facere oportet“ gerichtet, sie sich mithin nicht auf einen bestimmten Gegenstand, sondern bezog auf „all das, was der Beklagte dem Kläger zu leisten hat“, so wurde der Umfang der Kondemnation anhand dessen ermittelt, „quod (bzw. quanti) interest“. ${ }^{116}$ Jenseits dieser flexiblen Formel zur Schadensschätzung gab es weder einen Interesse-Terminus noch einen dogmatisch durchformten Interesse-Begriff. Es handelte sich nach römischem Verständnis weniger um eine rechtlichen denn um eine tatsächliche Frage: Quatenus cuius intersit, in facto, non in iure consistit (Paulus D. 50.17.24).

Es finden sich in den Digesten zahlreiche, mitunter widersprüchliche Fragmente, die sich durchaus dunkel zu schwierigen Fragen wie der Ersatzfähigkeit von entgangenem Gewinn oder Mangelfolgeschäden äußern. Die mittelalterlichen Zivilisten sahen sich nun der Herausforderung gegenüber, aus dieser Quellenlage eine kohärente Theorie zu konstruieren. Sie entwickelten eine Doktrin so reich an subtilen Distinktionen, dass die Interessedogmatik später als eine der verschlungensten und überzüchtetsten Bereiche des Zivilrechts überhaupt galt. Im 17. Jahrhundert warnte etwas Sigismund Scaccia, dass die Interessenlehre ein riesiges Meer sei, das nur wenige gefahrlos beführen. ${ }^{117}$

An dieser Stelle kann die dorthin führende Entwicklung freilich nur in allergröbsten Umrissen geschildert werden. Die Entwicklung der Interessendogmatik hat Hans Jo-

112 Ausführlich M. Kaser/K. Hackl, Das römische Zivilprozessrecht, 2. Aufl., München 1997, $\$ \$ 22$ ff.

113 Gaius IV, 48; M. Kaser/R. Knütel, Römisches Privatrecht, 19. Aufl., München 2008 \$35, Rn. 1 ff.

114 Kaufklagen sind bonae fidei iudiciae, siehe M. Kaser, Das Römische Privatrecht, Erster Abschnitt, 2. Aufl., München 1971, $\mathbb{1 1 4}$, S. 486; $\mathbb{1 3 1}$, S. $556 \mathrm{ff}$.

115 Dazu etwa D. Medicus, Id quod interest, Köln 1962, S. 29 ff.

116 H. Honsell, Quod interest im bonae-fidei-iudicium, München 1969, S. 1 ff. Richtete sich die formula hingegen auf ein certum, so war der Standard „quanti ea res est”: Kaser/Knütel, Römisches Privatrecht (Fn. 113) $\$ 35, \mathrm{Rn} .7 \mathrm{f}$.

117 S. Scaccia, Tractatus de commerciis et cambio, Genevae 1664, $\mathbb{S}$ VII Gl. II n. 18, S. 443 f.: „mare amplissimum, in quo panci sine periculo navigarunt". 
sef Wieling monographisch auf das Anschaulichste nachvollzogen. ${ }^{118}$ Für uns ist vor allem ein Blick auf die Grundzüge der methodischen Schrittfolge von Nutzen. Zunächst musste die flexible Schätzformel des „quod interest" konzeptuell handhabbar gemacht werden. Dies ist der erste Schritt der Methode der distinctio, eine Induktion bzw. Synthese: ${ }^{119}$ Die ursprüngliche römische Wendung wurde hierbei zum Neologismus des „interesse“ substantiviert, ${ }^{120}$ und dabei ein Begriff geschaffen, der das „id quod interest" gleichsam reifiziert und be-greifbar macht. Dieser Begriff wird nun an die Spitze einer Begriffspyramide gestellt. In einem nächsten - deduktiven oder analytischen ${ }^{121}$ - Schritt werden Unterbegriffe abgeleitet, die allerdings ihrerseits wiederum durch Induktion aus den Quellen gewonnen werden. $\mathrm{Zu}$ den wohl wichtigsten und einflussreichsten Distinktionen gehörte zunächst die bekannte, auf einer justinianischen Konstitution (C. 7.47) beruhende Unterscheidung zwischen damnum emergens und lucrum cessans (vgl. heute etwa Art. 1149 Code Napoléon), und die die Schadensschätzung betreffende Distinktion von interesse singulare, commune und conventum. ${ }^{122}$

Besonders nachhaltig war die Wirkung der Kategorienbildung von interesse circa rem und interesse extra rem, mit denen etwa gar noch Pothier hantierte. ${ }^{123}$ Die Funktion der Distinktion bestand darin, die Reichweite der Schadensersatzpflicht zu begrenzen, indem wiederum ersatzfähige und nicht ersatzfähige Posten begrifflichen Kategorien zugeordnet wurden: Das interesse extra rem war die Konzeptualisierung zu entfernter und daher nicht ersatzfähiger Schäden. ${ }^{124}$ Bemerkenswert ist für uns wiederum, dass diese äußerst einflussreiche Entwicklung abstrakter Kategorien aus einem Digestenfragment extrapoliert wird, in dem weder das „interesse“ noch, naturgemäß, die Unterkategorien von „interesse extra rem“ und „circa rem“ Erwähnung finden:

118 H.J. Wieling, Interesse und Privatstrafe vom Mittelalter zum Bürgerlichen Gesetzbuch, Wien 1970, S. 81 ff.; M.J. Schermaier, in: M. Schmoeckel/J. Rückert/R. Zimmermann (Hrsg.), Historisch-kritischer Kommentar zum BGB, Band II - Allgemeines Schuldrecht, Tübingen 2007, $\$ \mathbb{S} 280-285$, Rn. 52.

119 H. Kantorowicz, Studies in the Glossators of the Roman Law - Newly Discovered Writings of the Twelfth Century (in Zusammenarbeit mit W.W. Buckland), Cambridge University Press 1938, S. 215; Meyer, Distinktionstechnik (Fn. 42), S. 82.

120 Zur Etymologie und historischer Semantik des Interesse-Begriffs N. Lubmann, Interesse und Interessenjurisprudenz im Spannungsfeld von Gesetzgebung und Rechtsprechung, Zeitschrift für Neuere Rechtsgeschichte 11 (1990), S. 1 (6 ff.).

121 Meyer, Distinktionstechnik (Fn. 42), S. 82.

122 D. 9.2.33pr.; D. 35.2.63pr.; D. 19.1.1pr.

123 R.-J. Pothier, Traité du Contrat de Vente, Band 1, Paris 1823, Partie II, Chapitre I, Sec. I, Article V, $\mathbb{S}$ V, N. 71.

124 H. Lange, Schadensersatz und Privatstrafe in der mittelalterlichen Rechtstheorie, Wien 1955, S. 20; M.J. Schermaier, in: HKK (Fn. 118) $\$ \$ \$ 280-285$ Rn. 51; R. Zimmermann, The Law of Obligations, Oxford University Press 1996, S. 830 ff. 
D. 19.1.21.3 Paulus libro 33 ad edictum

Cum per venditorem steterit, quo minus rem tradat, omnis utilitas emptoris in aestimationem venit, quae modo circa ipsam rem consistit: neque enim si potuit ex vino puta negotiari et lucrum facere, id aestimandum est, non magis quam si triticum emerit et ob eam rem, quod non sit traditum, familia eius fame laboraverit: nam pretium tritici, non servorum fame necatorum consequitur. (...)

Wenn es am Verkäufer liegt, dass er die Sache nicht übergibt, geht der gesamte Schaden des Käufers in die Schätzung ein, sofern dieser sich nur auf die Sache selbst bezieht. Es ist nämlich nicht in die Schätzung einzubeziehen, wenn der Käufer mit dem Wein etwa Handel treiben und Gewinn erzielen könnte; ebenso wenig wie wenn er Getreide gekauft hat und seine Sklaven deswegen, weil es nicht übergeben worden ist, Hunger leiden; der Käufer erlangt nur den Wert des Getreides, nicht den Wert der verhungerten Sklaven.

Aus der untechnischen Formulierung des Paulus von der utilitas circa rem konstruierten die Glossatoren die Kategorie des interesse circa rem. Dessen „Existenz “ freilich gebot die Erfindung eines Gegenstücks, des interesse extra rem. Diese dergestalt den Quellen abgewonnenen Kategorien konnten in einer Begriffspyramide auf wechselnden Hierarchieebenen miteinander kombiniert und rekombiniert werden. ${ }^{125} \mathrm{Zu}$ dem traten weitere, immer subtilere Distinktionen hinzu.

Wohin die Lust an der kabbalistischen Permutation, an der Hinzuerfindung immer neuer Kategorien führen kann - das populärste Beispiel dafür ist der „Arbor super interesse" des französischen Rechtslehrers Petrus Rebuffus (1487-1557), ein Baumdiagramm, das die Distinktionen aus dessen Traktat über das Interesse visualisiert. ${ }^{126}$ Nicht weniger als 48 verschiedene Variationen des Interesses prangen an diesem Baum, „zum Teil in der wunderlichsten Weise erfundene Rubriken“, wie Endemann formulierte. ${ }^{127}$ Dogmatischer Wildwuchs im wahrsten Sinne des Wortes, und eine Taxonomie nicht weniger grotesk als die Borges’ „gewisser chinesischer Enzyklopaedie“. Rebuffus freilich schrieb in der „Neuzeit“ und nicht im Mittelalter: Von 1529 bis 1534 lehrte er an der Seite des Alciatus in Bourges und hatte dann als Kollege des Duarenus - einen kanonistischen Lehrstuhl in Paris inne; auch im „humanistischen“ Frankreich verhinderte eine derart zur Karikatur gewordene scholastische Methode offenbar nicht eine glänzende akademische Karriere. ${ }^{128} \mathrm{Im}$

125 Wieling, Interesse (Fn. 118), S. 32 ff.

126 Codicis DN. Iustiniani (...) libri IX priores, Tomus Quartus, Venedig,Apud Iuntas 1592, Spalte 2129-30 recto. Siehe Abbildung 1.

127 W. Endemann, Studien in der Romanisch-Kanonistischen Wirtschafts- und Rechtslehre bis gegen Ende des 17. Jahrhunderts, Band 2, Berlin 1883, Neudruck, Aalen 1962, S. 250.

128 R. Chabanne, Rebuffe, in: R. Naz (Hrsg.), Dictionnaire du Droit Canonique, Band VII, Paris: Librairie Letouzey et Ané 1965, S.475; E. Holthöfer, Rebuffi, Pierre, in: M. Stolleis (Hrsg.), Juristen Ein biographisches Lexikon, München 1995, S. 513. 
„Baum“ des Rebuffus manifestiert sich das Verlangen nach totaler manifestatio. Er häuft Distinktionen, die zum Harmonisieren der Quellen längst nicht mehr notwendig sind. Diese wildwuchernde Dogmatik hat ihre Verbindung zu den Wurzeln in der Schul-Rationalität der Methode längst verloren. Der „mentale Habitus“, seiner funktionalen Implikationen entkleidet, ergeht sich nurmehr in einer zweckfreien Ästhetik: hier, tatsächlich, l'art pour l'art.

\section{Bartolus: Formvollendung und „Zweckrationalität“}

Die Interessedogmatik liefert aber auch umgekehrt Anschauungsmaterial dafür, wie im Rahmen der bewussten wie unbewussten Vorgaben des „mentalen Habitus“ Form und Inhalt eine glücklichere, synergetische Beziehung eingehen. Die Evolution der Stilistik geht zugleich einher mit einer fortschreitenden Emanzipation vom Text, was zunehmend die Berücksichtigung nicht text-immanenter Zweckerwägungen erlaubt, ohne formaliter den Textbezug aufgeben zu müssen. Eine Entwicklung gleich derer, die Knut Wolfgang Nörr in anderem Zusammenhang als Fortschreiten von der „Textrationalität zur Zweckrationalität“ bezeichnet hat. ${ }^{129}$ Fündig werden wir vor allem bei Bartolus, Meister scholastischer Stilistik und zugleich vor allem brillanter - und ausgepichter - Jurist. ${ }^{130}$

Auch hier muss sich die Darstellung aus Platzgründen auf ein bündig dargestelltes Beispiel beschränken. Wir hatten schon gesehen, dass aus dem Paulus-Fragment D. 19.1.21.3 die Distinktion zwischen interesse circa rem und extra rem gewonnen wurde. Andere Fragmente mussten in diese - den Quellentexten ja fremde - Dichotomie eingepasst werden. So etwa die bekannte Ulpian-Stelle D. 19.1.13 pr., die den Ersatz von Mangelfolgeschäden zuspricht, wenn der Verkäufer wissentlich schlecht leistet (etwa eine krankes Stück Vieh oder marodes Bauholz liefert): Dies konnte gleichwohl begrifflich als interesse extra rem aufgefasst werden, in Konsonanz mit D. 19.1.21.3, da u.a. mit der Arglist des Schuldners ein distinguierendes Merkmal gegeben war - einer der Quellen der sog. Schuldstufenlehre, die sich im deutschen gemeinen Recht bis in das 19. Jahrhundert hinein hielt. ${ }^{131}$

Die Dichotomie von circalextra rem lag jedoch quer zu der von damnum und lucrum (entgangenem Gewinn): Hieß es doch im Codex (C. 7.47), dass das „id quod interest" sich aus beidem zusammensetze. In D. 19.1.21.3 unterscheidet Paulus jedoch nicht zwischen entgangenem Gewinn und Folgeschäden und hält beide nicht für ersatzfähig. Ist das lucrum mithin ein „interesse circa rem “ und daher in der Regel (z.B. bei Abwesenheit von Arglist) nicht ersatzfähig? Dem widerspricht wiederum ein an-

129 Nörr (Fn. 36) S. $24 \mathrm{f}$.

130 Zur Wirkung und Rezeption Bartolus'scher Dogmatik im Bereich der Interessendogmatik siehe noch stets H. Lange, Bartolus' Einfluss auf die Entwicklung des Schadensersatzrechts, in: Bartolo da Sassoferrato, Studi e documenti per il VI centenario, Bd. 2, Mailand: Giuffrè 1962, S. $281 \mathrm{ff}$.

131 H. Dedek, Negative Haftung aus Vertrag, Tübingen 2006, S. 135-144/148 f. 
deres Ulpian-Fragment (D. 13.4.2.8): Hier wird das lucrum zugesprochen, ohne dass die Kriterien Erwähnung fänden, die zur Distinktion zwischen D. 19.1.21.3 und D. 19.1.13 pr. herangezogen werden konnten; Ulpian erwähnt nur den Umstand, dass der geschädigte Gläubiger Handel zu treiben pflegte.

Die Accursische Glosse macht sich die Auflösung dieses Spannungsverhältnisses noch recht einfach: In der gl. „Aestimandum “132 zu D. 19.1.21.3 wird schlicht ausgeführt, dass das lucrum in der Regel nicht zu ersetzen sei; allein von der Regel gebe es zahlreiche Ausnahmen („Sed specalia sunt multa contra dictam regulam“), worauf eine Aufzählung aller Stellen folgt, die nicht recht zur Regel passen wollen. Dies ist freilich eine simple Manier, die Stellen in Einklang zu bringen; solcher Mangel an Eleganz und Originalität mag Roger Bacon vorgeschwebt haben, als er sprach von den concordiae violentes, den erzwungenen Konkordanzen, wie sie die Juristen gebrauchten. ${ }^{133}$

Ganz anders die rund ein Jahrhundert jüngere, „hochscholastische“ Stellungnahme des Bartolus in dessen Kodexkommentar. Die komplexe Problematik, die Ersetzbarkeit des lucrum, hebt er sich für den Schluss seiner Ausführungen zu den Kategorien des Interesse auf. ${ }^{134}$ Die Spannung zwischen den Quellen löst er auf, indem er den Begriff des lucrum durch fünf Subdistinktionen weiter unterteilt. Die starke, fünffache Anapher „Quoddam est lucrum “135 hebt die Subtilität der Distinktion hervor: Mit jeder weiteren Wiederholung wird erneut betont, dass man die Unterscheidungskunst eben noch ein wenig weiter vorantreiben könne, und dass sich zugleich der intellektuelle Widerspruch auflöse, wenn nur das Wesen des jeweils einschlägigen Typs von lucrum richtig verstanden und erklärt werde.

Bartolus vermeidet hierbei die schlichte Gleichsetzung von lucrum und interesse extra rem und führt stattdessen die Beurteilung der Ersatzfähigkeit jeweils auf allgemeine Prinzipien zurück. So unterscheidet er auch zwischen einem lucrum, in dem die „Natur“ einer Sache zum Ausdruck komme, wie etwa Früchten; und einem lucrum, in dem menschliche „industria“ sich äußere. Da es sich in letzterem Falle nicht um den „natürlichen“ Lauf der Dinge handelt und dementsprechend die Unberechenbarkeit menschlichen Handelns als Unsicherheitsfaktor hinzutritt, unterscheidet Bartolus

132 S. 1232 der verwendeten Ausgabe: DIGESTUM VETUS SEV PANDECTARUM IURIS CIVILIS, TOMUS PRIMUS, CVM PANDECTIS FLORENTINIS, Venedig: Editio Postrema 1575.

133 Roger Bacon, Opus Minus, Fr. Rogeri Bacon Opera quaedam hactenus inedita, Vol. 1, hrsg. von J.S. Brewer, London: Longman, Green, Longman and Roberts 1859, S. 323. Wohl bei einer späteren Bearbeitung hat Accursius dann den Zusatz „Sed hodie secus, ut C 7,47 l.un. “ beigefügt, mithin wohl den Widerspruch darin aufgelöst gesehen, dass die justinianische Konstitution die widersprechenden Digestenstellen aufhebe; dazu H. Lange, Schadensersatz und Privatstrafe in der mittelalterlichen Rechtstheorie, Münster, Köln, S. 37; ders., Bartolus (Fn. 130), S. 287.

134 Bartoli a Saxoferrato, Omnium Iuris Interpretum Antesignani Commentaria. Tomus Octavus, In secundam, atque Tertiam Codicis Partem, Venedig 1590, fol. 71, verso (linke Spalte), n. 22-25; danach geht er zur Diskussion der Duplum-Grenze über.

135 Siehe Abbildung 2. 
folgerichtig weiter danach, ob das hypothetische Erwirtschaften des Gewinns nicht als zu unsicher gelten müsse (n. 25). Wenn sich jemand als Kaufmann betätige, sei der Gewinn sicher - wobei er auf D. 13.4.2.8 verweist; fehle es an solcher Professionalität, so sei der entgangene Gewinn „incertum“ - was wiederum die Entscheidung in D. 19.1.21.3 erklärt, wo vom gewohnheits- oder berufsmäßigen Handeltreiben keine Rede ist.

Damit ist mit feinster scholastistischer Ziselierkunst concordantia hergestellt, bewiesen, dass beide Quellen „diversa, sed non adversa“ waren. Zugleich ist aber auch eine Erklärung gefunden, die - im besten Dworkin'schen Sinne ${ }^{136}$ - das Recht „rationalisiert“, indem sie die für unverbundenen Aussagen autoritativer Rechtsquellen (Gerichtsurteile für Dworkin, die „leges“ des Corpus Iuris bei Bartolus) eine Erklärung findet, die sowohl passt (,fits“) als sie auch rational rechtfertigt (,justifies“). Die „Zweckrationalität“ und Lebensnähe der Argumentation erschließt sich uns ohne weiteres: Sowohl im Hinblick auf die Bedeutung der Handels im historischen Kontext der oberitalienischen Städtekultur, als auch auf die Allgemeingültigkeit der herangezogenen Begründung, die mit der Wahrscheinlichkeit des Schadenseintritts als ausschlaggebendem Kriterium moderne Wertungsprinzipien der Schadensermittlung vorwegnimmt. ${ }^{137}$

\section{E. Schluss}

Kehren wir zurück zu dem Anfang unserer Untersuchung. Die gängige Art und Weise, die Moderne als Triumph der Vernunft zu denken, ${ }^{138}$ hat zum einen dazu geführt, sich der Vor-Moderne zu entfremden, ja deren intellektuelle Errungenschaften herabzusetzen; zum anderen dazu, die irrationalen, ja mythischen Seiten gerade dieser Vernunftstradition selbst in ihrer Bedeutung traditionell zu unterschätzen. ${ }^{139}$ Die Erkenntnis, dass auch unsere Vorstellung von rationaler Entscheidungsfindung und Wissenschaftlichkeit einer Tradition, einer Entwicklung entspringt, ermöglicht uns das Verständnis andersartiger Rationalitäten.

136 R. Dworkin, Law's Ambition for Itself, Virginia Law Review 71 (1985), S. 173 (177: „fit“ und 178: ,justification“).

137 Die Zurückhaltung gegenüber dem Ersatz von entgangenem Gewinn hielt sich freilich auch nach Bartolus. Der entscheidende gedankliche Fortschritt wird gemeinhin Dumoulin und seiner Theorie der Vorhersehbarkeit zugeschrieben, die dann von Pothier übernommen wurde und beeinflusste auf diesem Wege sogar das common law, vgl. A. W.B. Simpson, Innovation in Nineteenth Century Contract Law, Law Quarterly Review 91 (1975) S. 247 (274 ff.). Zur Würdigung des nachhaltigen Einflusses des Bartolus nochmals Lange, Bartolus (Fn. 130) S. 284-288.

138 Kritisch zu dieser Lesart der (eingangs erwähnten) Schriften Max Webers vgl. A. Sica, Rationalization and Culture, in: S. Turner (Hrsg.), The Cambridge Companion to Weber, Cambridge University Press 2000, S. 42 (47 ff.).

139 M. Horkheimer/T. Adorno, Dialektik der Aufklärung, Philosophische Fragmente, Frankfurt 1981, S. 13 ff. ff. Zum Spannungsverhältnis zwischen der notwendigen Irrationalität traditionalen Verhaltens und dem Selbstverständnis einer Tradition als Tradition von Rationalität siehe bereits oben zu Fn. 10. 
Dabei hält uns der Blick auf die mittelalterlichen Scholastiker den Spiegel vor. Deren ratio geht von Axiomen aus, die wir nicht nachvollziehen können; doch auch wir akzeptieren letzte Wahrheiten, die Geltung einer Grundnorm, als schlicht „gesetzt“. Wir sehen bei den Scholastikern eben jene ratio am Werk, aber ebenso irrationale, unbewusste oder vorbewusste Faktoren; die Scholastik ist viel mehr als nur Methode, sie ist umfassender „mentaler Habitus“, der sich auch in einer besonderen Stilistik, wenn man so will: einer gewissen „Ästhetik“ äußert.

Es ist kein Zufall, dass Pierre Bourdieu die Figur des „Habitus“ in Anlehnung an Panofskys Beschreibung der Scholastik als „mental habit“ entwickelte, um daran die nachhaltige Wirkung von intellektueller Sozialisierung durch Bildungsinstitutionen zu exemplifizieren. ${ }^{140}$ Niemals wieder war der Zusammenhang, ja die Einheit zwischen Ausbildung, Denkungsart und unbewusstem „Habitus“ so greifbar wie in der Scholastik, der „Wissenschaft in der Gestalt der Schule“. Dies illustriert jedoch nur besonders lebhaft das Prinzip, das freilich auch heute Geltung beansprucht: dass nicht nur die scholastische „Schule“, sondern auch die moderne Rechts-Schule, die „law school“, nicht nur Inhalte vermittelt, sondern auch einen „Habitus“ erzeugt.

Wird man der so gearteten Wirkmechanismen gewärtig, so verlieren Versuche, diesen unreflektierten, nicht-rationalisierten Ausschnitt der Rechtswirklichkeit zu beschreiben, ihren „irrationalen“, vielleicht gar seltsamen Beiklang. Solche Selbsterkenntnis erleichtert das Verständnis der Rechtsgeschichte ebenso wie das anderer Rechtskulturen; nicht zuletzt aber auch das der alltäglichen Praxis der Rechtsanwendung. 


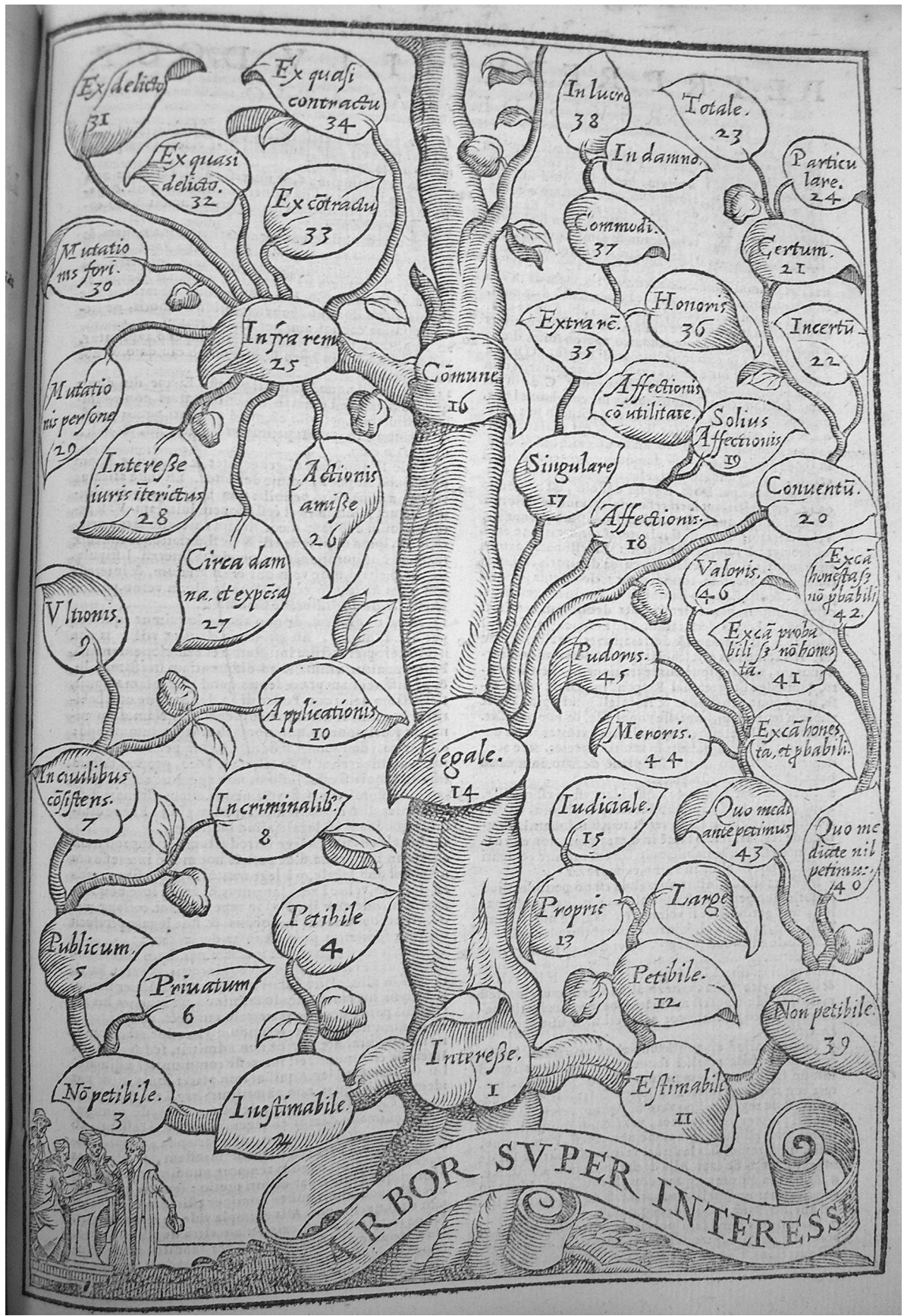

Abbildung 1: Codicis DN. Iustiniani (...) libri IX priores, Tomus Quartus, Venedig, Apud Iuntas, 1592, Spalte 2129-30 recto 


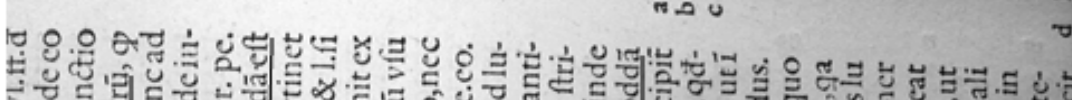
5. 은 के त.

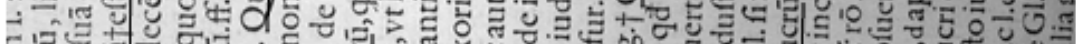
톰

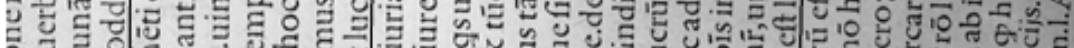
范 范

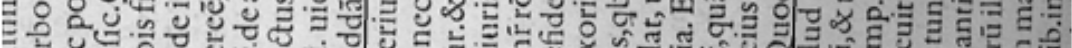

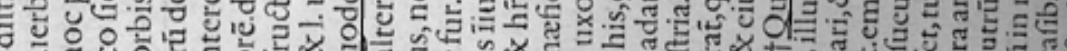

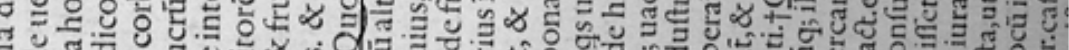

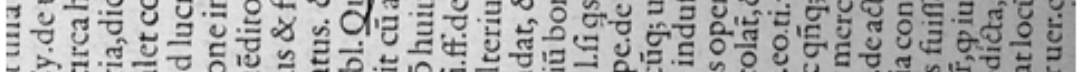

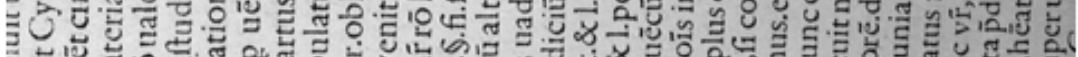

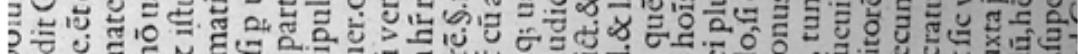

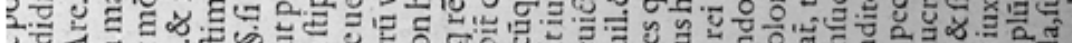
万ृ兀

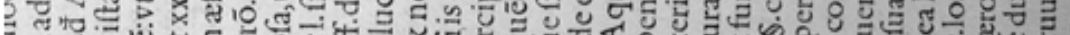

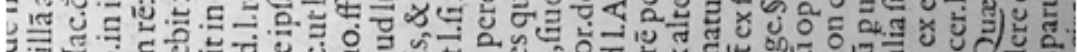
y

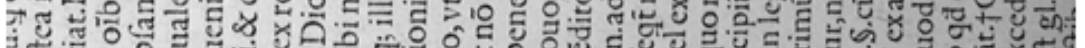

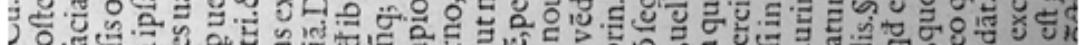

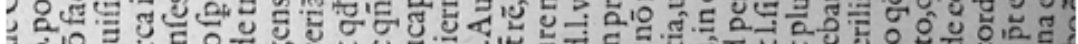

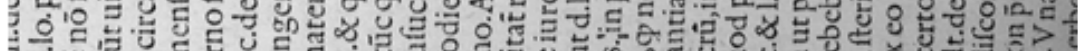

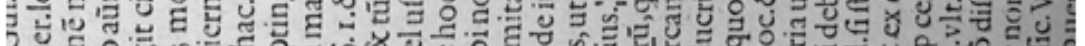

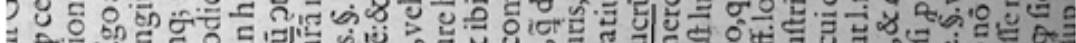

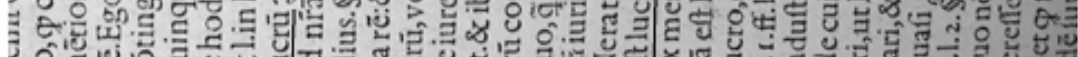

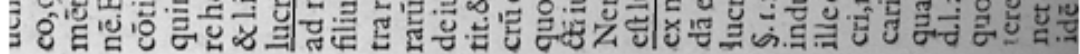

$$
\text { ส } \pi \text { ก }
$$

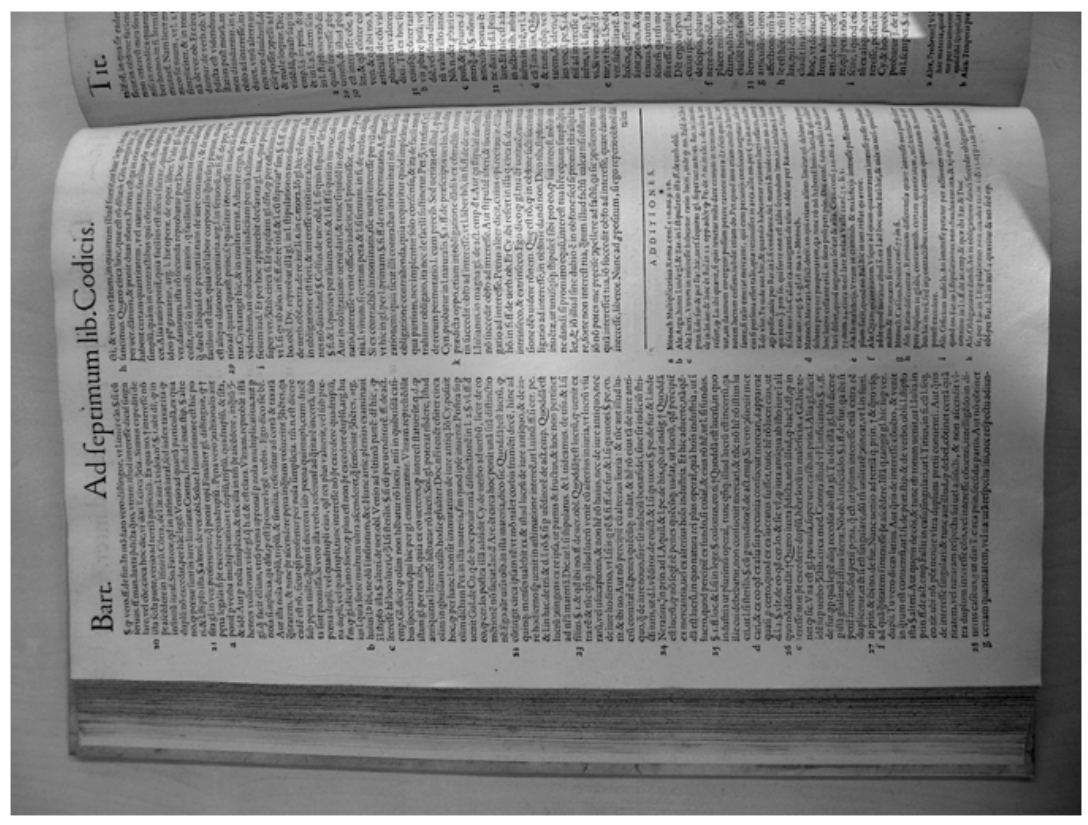

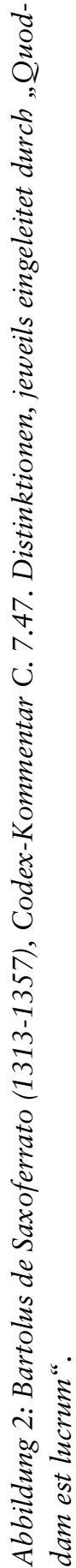

